\title{
The Nonlinear Steepest Descent Approach for Long Time Behavior of the Two-component Coupled Sasa-Satsuma Equation with a $5 \times 5$ Lax Pair
}

\author{
Xiu-Bin Wang* and Bo Han
}

\begin{abstract}
Under investigation in this work is the coupled Sasa-Satsuma equation, which can describe the propagations of two optical pulse envelopes in birefringent fibers. The Riemann-Hilbert problem for the equation is formulated on the basis of the corresponding $5 \times 5$ matrix spectral problem, which allows us to present a suitable representation for the solution of the equation. Then the Deift-Zhou steepest descent method is used to analyze the long time behavior of the coupled Sasa-Satsuma equation.
\end{abstract}

\section{Introduction}

It is well-known that the standard nonlinear Schrödinger (NLS) equation is a key integrable system in the field of mathematical physics. There are many physical phenomenon where the NLS equation appears. For instance, the NLS equation describes slowly varying wave envelopes in dispersive media from water waves, nonlinear optics, and plasma physics. In particular, the NLS equation can be used to model the soliton propagation in optical fibers where only the self-phase modulation effects and the group velocity dispersion are discussed. However, for ultrashort pulse in optical fibers, the effects of the self steepening, the third-order dispersion, and the stimulated Raman scattering should be taken into account. Because of these effects, the dynamic behaviors of the ultrashort pulses can be described by the higher-order NLS equation (also called Sasa-Satsuma equation) 25, 26, $39,43,45$

$$
q_{T}+\frac{1}{2} q_{X X}+|q|^{2} q+i \epsilon\left\{q_{X X X}+6|q|^{2} q_{X}+3 q\left(|q|^{2}\right)_{X}\right\}=0,
$$

where $q=q(X, T)$ is a complex-valued function. In addition, to model the propagations of two optical pulse envelopes in birefringent fibers well, some coupled Sasa-Satsuma equations were proposed and discussed $23,24,30,38$. In this work, we therefore focus on

Received June 15, 2020; Accepted August 26, 2020.

Communicated by Jenn-Nan Wang.

2010 Mathematics Subject Classification. 35Q51, 35Q53, 35C99, 68W30, 74J35.

Key words and phrases. the coupled Sasa-Satsuma (CSS) equation, the Deift-Zhou steepest descent method, long time asymptotics, Riemann-Hilbert problem (RHP).

*Corresponding author. 
a coupled Sasa-Satsuma (CSS in brief) equation

$$
\begin{aligned}
& i q_{1 T}+\frac{1}{2} q_{1 X X}+\left(\left|q_{1}\right|^{2}+\left|q_{2}\right|^{2}\right) q_{1} \\
+ & i \epsilon\left\{q_{1 X X X}+6\left(\left|q_{1}\right|^{2}+\left|q_{2}\right|^{2}\right) q_{1 X}+3 q_{1}\left(\left|q_{1}\right|^{2}+\left|q_{2}\right|^{2}\right)_{X}\right\}=0, \\
& i q_{2 T}+\frac{1}{2} q_{2 X X}+\left(\left|q_{1}\right|^{2}+\left|q_{2}\right|^{2}\right) q_{2} \\
+ & i \epsilon\left\{q_{2 X X X}+6\left(\left|q_{1}\right|^{2}+\left|q_{2}\right|^{2}\right) q_{2 X}+3 q_{2}\left(\left|q_{1}\right|^{2}+\left|q_{2}\right|^{2}\right)_{X}\right\}=0,
\end{aligned}
$$

which can be rewritten in the following form 24]

$$
\begin{gathered}
u_{t}+\epsilon\left\{u_{x x x}+6\left(|u|^{2}+|v|^{2}\right) u_{x}+3 u\left(|u|^{2}+|v|^{2}\right)_{x}\right\}=0, \\
v_{t}+\epsilon\left\{v_{x x x}+6\left(|u|^{2}+|v|^{2}\right) v_{x}+3 v\left(|u|^{2}+|v|^{2}\right)_{x}\right\}=0, \\
u(x, 0)=u_{0}(x), \quad v(x, 0)=v_{0}(x),
\end{gathered}
$$

by introducing the gauge, Galilean and scale transformations

$$
\begin{aligned}
u(x, t) & =q_{1}(X, T) \exp \left[-\frac{i}{6 \epsilon}\left(X-\frac{T}{18 \epsilon}\right)\right], \\
v(x, t) & =q_{2}(X, T) \exp \left[-\frac{i}{6 \epsilon}\left(X-\frac{T}{18 \epsilon}\right)\right], \\
x & =X-\frac{T}{12 \epsilon}, \quad t=T,
\end{aligned}
$$

where $\left(u_{0}, v_{0}\right)$ lie in the Schwartz space, $\epsilon$ is the ratio of the width of the spectra to the carrier frequency, and the last three terms in the left-hand side of (1.1) stand for the thirdorder dispersion, self-steepening, and stimulated Raman scattering effects, respectively. Besides, $q_{1}=q_{1}(X, T)$ and $q_{2}=q_{2}(X, T)$ are two complex functions of variables $X, T$, The CSS equation 1.2 is still completely integrable. Additionally, the CSS equation 1.2 has also been investigated via Darboux transformation, Darboux-Bäcklund transformation and Hirota method etc. Recently, we have studied the long-time behavior and rogue wave solutions of the integrable three-component coupled nonlinear Schrödinger equation [33, 35. In this paper, we will consider the long-time asymptotics of the CSS equation 1.2) on the line. In the following, we let $\epsilon=1$ for the convenience of the analysis.

In recent years, there are many investigations on long time asymptotics and exact solutions of nonlinear evolution equations 8 10, 16, 19,21, 27, 28, 32, 34, 36, 41. It is also known that the Deift-Zhou steepest descent approach is a powerful approach to analyze the long time behavior for integrable nonlinear wave equations $4,7,11,14,17,18,22,29$, 31, 40, 42. However, since $(1.2)$ contains a $5 \times 5$ matrix spectral problem, the long time asymptotics for 1.2 is rather complicated to consider. The research in this direction, to the best of our knowledge, has not been conducted before. The main purpose of the present article is to analyze the long time asymptotics of $(1.2)$ by utilizing the Riemann-Hilbert problem (RHP) via the Deift-Zhou steepest descent method. 
The structure of this paper is given as follows. In Section 2 , we derive a $5 \times 5$ matrix RHP and find that the solution of $(1.2)$ can be given by the solution of this RHP. In Section 3, we obtain the main conclusion of this work by using the Deift-Zhou steepest descent method. Finally, the last section summarizes the main results of this article.

\section{Riemann-Hilbert problem}

System 1.2 is still completely integrable. Its Lax pair yields 24

$$
\begin{aligned}
& \psi_{x}(x, t ; \lambda)=i \lambda \sigma \psi(x, t ; \lambda)+\mathbf{U}(x, t ; \lambda) \psi(x, t ; \lambda), \\
& \psi_{t}(x, t ; \lambda)=4 i \lambda^{3} \sigma \psi(x, t ; \lambda)+\mathbf{V}(x, t ; \lambda) \psi(x, t ; \lambda),
\end{aligned}
$$

where

$$
\sigma=\left(\begin{array}{cc}
\mathcal{I}_{4 \times 4} & \mathbf{0} \\
\mathbf{0} & -1
\end{array}\right), \quad \mathbf{U}=\left(\begin{array}{cc}
\mathbf{0}_{4 \times 4} & \mathcal{U} \\
-\mathcal{U}^{\dagger} & 0
\end{array}\right), \quad \mathcal{U}=\left(\begin{array}{c}
\mathbf{u} \\
\mathbf{v}
\end{array}\right), \quad \mathbf{u}=\left(\begin{array}{l}
u \\
\bar{u}
\end{array}\right), \quad \mathbf{v}=\left(\begin{array}{l}
v \\
\bar{v}
\end{array}\right)
$$

with

$$
\mathbf{V}(x, t, \lambda)=4 \lambda^{2} \mathbf{U}-2 i \lambda \sigma\left(\mathbf{U}_{x}-\mathbf{U}^{2}\right)+\left(\mathbf{U}_{x} \mathbf{U}-\mathbf{U}_{x}\right)-\mathbf{U}_{x x}+2 \mathbf{U}^{3} .
$$

Here the overbar represents the complex conjugation and "†" represents Hermitian of a matrix.

In the following, introducing a new matrix function by

$$
\psi(x, t ; \lambda)=\mu(x, t ; \lambda) e^{i\left(\lambda x+4 \lambda^{3} t\right) \sigma}
$$

the spectral problem (2.1) then gives

$$
\begin{aligned}
\mu_{x}(x, t ; \lambda)-i \lambda[\sigma, \mu(x, t ; \lambda)] & =\mathbf{U}(x, t) \mu(x, t ; \lambda), \\
\mu_{t}(x, t ; \lambda)-4 i \lambda^{3}[\sigma, \mu(x, t ; \lambda)] & =\mathbf{V}(x, t ; \lambda) \mu(x, t ; \lambda) .
\end{aligned}
$$

We next present two eigenfunctions $\mu_{ \pm}(x, t ; \lambda)$ of $x$-part of $(2.2)$ by the following Volterra type integral equations

$$
\mu_{ \pm}=\mathcal{I}+\int_{ \pm \infty}^{x} e^{-i \lambda(x-\xi) \widehat{\sigma}}\left[\mathbf{U}(\xi, t) \mu_{ \pm}(\xi, t ; \lambda)\right] d \xi
$$

where $\widehat{\sigma}$ represents the operators which act on a $5 \times 5$ matrix $\Omega$ by $\widehat{\sigma}=[\sigma, \Omega]$. Here $e^{\widehat{\sigma}}=e^{\sigma} \Omega e^{\sigma}$. Then we rewrite $\mu_{ \pm}(x, t ; \lambda)$ as

$$
\mu_{ \pm}(x, t ; \lambda)=\left(\mu_{ \pm L}(x, t ; \lambda), \mu_{ \pm R}(x, t ; \lambda)\right)
$$


where the first fourth columns of $\mu_{ \pm}(x, t ; \lambda)$ and fifth column are expressed by $\mu_{ \pm}(x, t ; \lambda)$ and $\mu_{ \pm R}(x, t ; \lambda)$, respectively. From $(2.3)$, we know that $\mu_{+L}, \mu_{-R}$ and $\mu_{-L}, \mu_{+R}$ are analytic in $\mathbb{C}_{-}$and $\mathbb{C}_{+}$, respectively. Furthermore

$$
\begin{array}{ll}
\left(\mu_{+L}(x, t ; \lambda), \mu_{-R}(x, t ; \lambda)\right)=\mathcal{I}+O\left(\frac{1}{\lambda}\right), & \lambda \in \mathbb{C}_{-} \rightarrow \infty, \\
\left(\mu_{-L}(x, t ; \lambda), \mu_{+R}(x, t ; \lambda)\right)=\mathcal{I}+O\left(\frac{1}{\lambda}\right), & \lambda \in \mathbb{C}_{+} \rightarrow \infty .
\end{array}
$$

The solutions of the equation of differential equation 2.2 can be related by a matrix independent of $x$ and $t$. As a result

$$
\mu_{-}(x, t ; \lambda)=\mu_{+}(x, t ; \lambda) e^{i\left(\lambda x+4 i \lambda^{3} t\right) \widehat{\sigma}} s(\lambda) .
$$

Evaluation at $t=0$ arrives at

$$
s(\lambda)=\lim _{x \rightarrow+\infty} e^{-i \lambda x \widehat{\sigma}} \mu_{-}(x, 0 ; \lambda),
$$

i.e.,

$$
s(\lambda)=\mathcal{I}+\int_{-\infty}^{+\infty} e^{-i \lambda x \widehat{\sigma}}\left[\mathbf{U}(x, 0) \mu_{-}(x, 0 ; \lambda)\right] d x .
$$

The fact that $\operatorname{tr}(\mathbf{U})=0$ together with 2.3$)$ indicates

$$
\operatorname{det}\left(\mu_{ \pm}(x, t ; \lambda)\right)=1 \text {. }
$$

Therefore, we obtain

$$
\operatorname{det}(s(\lambda))=1
$$

Additionally, we know that

$$
\mathbf{U}^{\dagger}(x, t ; \bar{\lambda})=-\mathbf{U}(x, t ; \lambda), \quad \overline{\mathbf{U}(x, t ;-\bar{\lambda})}=\nabla \mathbf{U}(x, t ; \lambda) \nabla,
$$

where

$$
\nabla=\left(\begin{array}{lllll}
0 & 1 & 0 & 0 & 0 \\
1 & 0 & 0 & 0 & 0 \\
0 & 0 & 0 & 1 & 0 \\
0 & 0 & 1 & 0 & 0 \\
0 & 0 & 0 & 0 & 1
\end{array}\right) .
$$

Furthermore, it follows from (2.1) that

$$
\psi_{x}^{A}(x, t ; \lambda)=(i \lambda \sigma-\mathbf{U}(x, t))^{T} \psi^{A}(x, t ; \lambda)
$$


with $\psi^{A}(x, t ; \lambda)=\left(\psi^{-1}(x, t ; \lambda)\right)^{T}$, where the superscript ' $\mathrm{T}$ ' represents a matrix transpose. Consequently, we have

$$
\psi^{\dagger}(x, t ; \bar{\lambda})=\psi^{-1}(x, t ; \lambda), \quad \psi(x, t ; \lambda)=\nabla \overline{\psi(x, t ;-\bar{\lambda})} \nabla .
$$

These relations indicate that the eigenfunctions $\mu_{j}(x, t ; \lambda)$ meet

$$
\mu^{\dagger}(x, t ; \bar{\lambda})=\mu^{-1}(x, t ; \lambda), \quad \mu(x, t ; \lambda)=\nabla \overline{\mu(x, t ;-\bar{\lambda})} \nabla, \quad j=1,2,
$$

where ' $\uparrow$ ' represents the Hermitian conjugate. To sum up, the matrix-valued function $s(\lambda)$ admits the following symmetries

$$
s^{\dagger}(\bar{\lambda})=s^{-1}(\lambda), \quad s(-\lambda)=\nabla \overline{s(\bar{\lambda})} \nabla .
$$

In the following, we rewrite a $5 \times 5$ matrix $\mathbf{A}$ as a block form

$$
\mathbf{A}=\left(\begin{array}{ll}
\mathbf{A}_{11} & \mathbf{A}_{12} \\
\mathbf{A}_{21} & \mathbf{A}_{22}
\end{array}\right)
$$

where $\mathbf{A}_{11}$ is a $4 \times 4$ matrix and $\mathbf{A}_{22}$ is scalar. It follows from $2.4-2.13$ that

$$
\begin{aligned}
s_{22}^{\dagger}(\bar{\lambda}) & =\operatorname{det}\left(s_{11}(\lambda)\right), & s_{11}(\lambda) & =\sigma_{1} \bar{s}_{11}(-\bar{\lambda}) \sigma_{1}, \\
s_{12}^{\dagger}(\bar{\lambda}) & =-s_{21} \operatorname{adj}\left(s_{11}(\lambda)\right), & \bar{s}_{21}(-\bar{\lambda}) \sigma_{1} & =s_{21}(\lambda),
\end{aligned}
$$

where

$$
\sigma_{1}=\left(\begin{array}{llll}
0 & 1 & 0 & 0 \\
1 & 0 & 0 & 0 \\
0 & 0 & 0 & 1 \\
0 & 0 & 1 & 0
\end{array}\right)
$$

and $\operatorname{adj}(\mathbf{B})$ represents the adjoint matrix of matrix $\mathbf{B}$. Because of the above expression (2.14), we rewrite $s(\lambda)$ as

$$
s(\lambda)=\left(\begin{array}{cc}
a(\lambda) & -\operatorname{adj}\left(a^{\dagger}(\bar{\lambda})\right) b^{\dagger}(\bar{\lambda}) \\
b(\lambda) & \operatorname{det}\left(a^{\dagger}(\bar{\lambda})\right)
\end{array}\right),
$$

where

$$
a(\lambda)=\sigma_{1} \bar{a}(-\bar{\lambda}) \sigma_{1}, \quad \bar{b}(-\bar{\lambda}) \sigma_{1}=b(\lambda) .
$$

It follows that $a(\lambda)$ and $b(\lambda)$ satisfy

$$
\begin{aligned}
& a(\lambda)=\mathcal{I}+\int_{-\infty}^{+\infty} \mathcal{U}(x, 0) \mu_{-, 21}(x, 0 ; \lambda) d x \\
& b(\lambda)=-\int_{-\infty}^{+\infty} e^{-2 i x \lambda} \mathcal{U}^{\dagger}(x, 0) \mu_{-, 11}(x, 0 ; \lambda) d x .
\end{aligned}
$$


Obviously, $a(\lambda)$ is analytic in $\mathbb{C}_{+}$.

Suppose that $\operatorname{det}(a(\lambda))$ admits $4 N$ simple zeros $\lambda_{1}, \ldots, \lambda_{4 N}$ in $\mathbb{C}_{+}$, where $\lambda_{N+j}=-\bar{\lambda}_{j}$, $j=1,2, \ldots 2 N$. Define

$$
M(\xi ; \lambda)= \begin{cases}\left(\mu_{-L}(\lambda) a^{-1}(\lambda), \mu_{+R}(\lambda)\right), & \lambda \in \mathbb{C}_{+} \\ \left(\mu_{+L}(\lambda), \mu_{-R}(\lambda) / \operatorname{det} a^{\dagger}(\bar{\lambda})\right), & \lambda \in \mathbb{C}_{-} .\end{cases}
$$

Theorem 2.1. Let $a(\lambda)$ and $b(\lambda)$ be determined by (2.15). Then $M(x, t ; \lambda)$ given by 2.16) satisfies the following matrix RHP. We find a meromorphic function $M(x, t ; \lambda)$ with simple poles at $\left\{\lambda_{j}\right\}_{1}^{4 N}$ and $\left\{\bar{\lambda}_{j}\right\}_{1}^{4 N}$, then it admits

$$
\begin{cases}M_{+}(\lambda)=M_{-}(\lambda) J(\lambda), & \lambda \in \mathbb{R} \\ M(\lambda)=\mathcal{I}+O\left(\frac{1}{\lambda}\right), & \lambda \rightarrow \infty\end{cases}
$$

and residue conditions

$$
\begin{aligned}
& \operatorname{Res}_{\lambda_{j}} M(\lambda)=\lim _{\lambda \rightarrow \lambda_{j}} M(\lambda)\left(\begin{array}{cc}
0 & 0 \\
\frac{e^{-2 i \theta(\lambda) t} b(\lambda) \operatorname{adj}(a(\lambda))}{\operatorname{det}(a(\lambda))} & 0
\end{array}\right), \\
& \operatorname{Res}_{\bar{\lambda}_{j}} M(\lambda)=\lim _{\lambda \rightarrow \bar{\lambda}_{j}} M(\lambda)\left(\begin{array}{cc}
0 & -\frac{e^{2 i \theta(\lambda) t} \operatorname{adj}\left(a^{\dagger}(\bar{\lambda})\right) b(\bar{\lambda})^{\dagger}}{\operatorname{det}\left(a^{\dagger}(\bar{\lambda})\right)} \\
0 & 0
\end{array}\right),
\end{aligned}
$$

where $j=1,2, \ldots, 2 N$, and

$$
\begin{gathered}
M_{ \pm}=\lim _{\epsilon \rightarrow 0^{+}} M(\lambda \pm i \epsilon), \quad \gamma(\lambda)=b(\lambda) a^{-1}(\lambda), \quad \lambda \in \mathbb{R} \\
J(\lambda)=\left(\begin{array}{cc}
\mathcal{I}+\gamma^{\dagger}(\bar{\lambda}) \gamma(\lambda) & e^{2 i \theta t} \gamma^{\dagger}(\bar{\lambda}) \\
e^{-2 i \theta t} \gamma(\lambda) & 1
\end{array}\right), \quad \theta=\lambda\left(\frac{x}{t}+4 \lambda^{2}\right) .
\end{gathered}
$$

Here $\gamma(\lambda)$ lies in Schwartz space and satisfies

$$
\gamma(\lambda)=\gamma^{\dagger}(-\bar{\lambda}) \sigma_{1}, \quad \sup _{\lambda \in \mathbb{R}} \gamma(\lambda)<\infty
$$

Let

$$
\mathcal{U}(x, t)=\left(\begin{array}{l}
\mathbf{u} \\
\mathbf{v}
\end{array}\right)=2 i \lim _{\lambda \rightarrow \infty}(\lambda M(x, t ; \lambda))_{12} .
$$

Then $u(x, t), v(x, t)$ represent the solution of the CSS equation (1.2).

\section{Long-time asymptotic analysis}

According to the idea of Deift and Zhou [6], we next consider the stationary points of the function $\theta$, i.e., setting $\frac{d \theta}{d \lambda}=0$, the stationary phase points are constructed for $x>0$ as $\pm \lambda_{0}= \pm \sqrt{\frac{x}{12 t}}$, Thus, $\theta=4 \lambda\left(\lambda^{2}-3 \lambda_{0}^{2}\right)$. In what follows, we mainly focus on physically interesting region $\lambda_{0} \in(0, C]$, where $C$ is a constant. 
3.1. Factorization of the jump matrix

We notice that the jump matrix admits two distinct factorizations

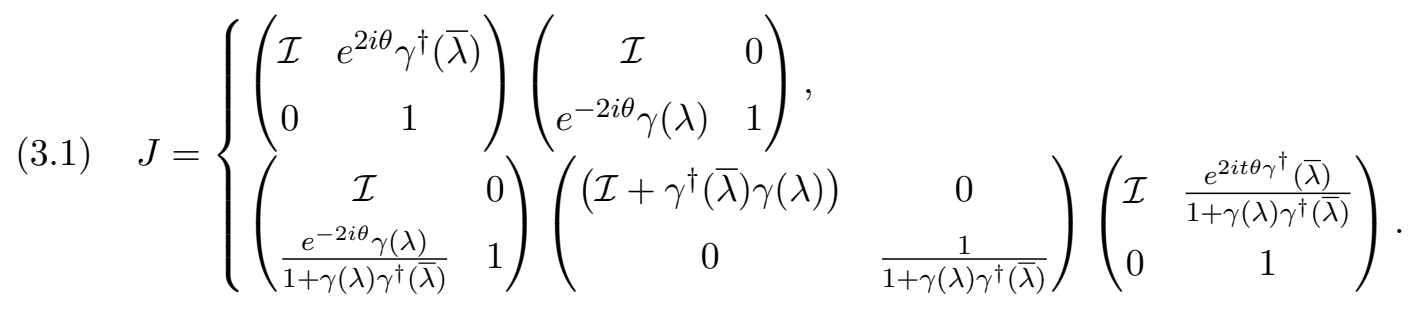

Next, we consider a function $\delta(\lambda)$ as the solution of the matrix problem

$$
\delta_{+}(\lambda)=\left\{\begin{array}{ll}
\left(\mathcal{I}+\gamma^{\dagger} \gamma\right) \delta_{-}(\lambda), & |\lambda|<\lambda_{0}, \\
\delta_{-}(\lambda), & |\lambda|>\lambda_{0}
\end{array} \quad \text { and } \quad \delta(\lambda) \rightarrow \mathcal{I}, \quad \lambda \rightarrow \infty\right.
$$

As the jump matrix $\left(\mathcal{I}+\gamma^{\dagger} \gamma\right)$ is positive definite, the vanishing lemma gives the existence and uniqueness of the function $\delta(\lambda)$. Moreover, we have

$$
\operatorname{det}\left(\delta_{+}(\lambda)\right)=\left\{\begin{array}{ll}
\left(1+|\gamma|^{2}\right) \operatorname{det}\left(\delta_{-}(\lambda)\right), & |\lambda|<\lambda_{0}, \\
\operatorname{det}\left(\delta_{-}(\lambda)\right), & |\lambda|>\lambda_{0},
\end{array} \quad \text { and } \quad \operatorname{det}(\delta(\lambda)) \rightarrow 1, \quad \lambda \rightarrow \infty\right.
$$

By utilizing the Plemelj formula [1], we get

$$
\operatorname{det}(\delta(\lambda))=\exp \left\{\frac{1}{2 \pi i} \int_{-\lambda_{0}}^{\lambda_{0}} \frac{\log \left(1+\gamma(\xi) \gamma^{\dagger}(\xi)\right)}{\xi-\lambda} d \xi\right\}=\left(\frac{\lambda+\lambda_{0}}{\lambda-\lambda_{0}}\right)^{i \nu} e^{\chi(\lambda)},
$$

where

$$
\begin{aligned}
\nu & =\frac{1}{2 \pi} \log \left(1+\gamma\left(\lambda_{0}\right) \gamma^{\dagger}\left(\lambda_{0}\right)\right)>0 \\
\chi(\lambda) & =\frac{1}{2 \pi i} \int_{-\lambda_{0}}^{\lambda_{0}} \log \left(\frac{1+\gamma(\xi) \gamma^{\dagger}(\xi)}{1+\gamma\left(\lambda_{0}\right) \gamma^{\dagger}\left(\lambda_{0}\right)}\right) \frac{d \xi}{\xi-\lambda} .
\end{aligned}
$$

Then we have used the following relation

$$
1+\gamma\left(\lambda_{0}\right) \gamma^{\dagger}\left(\lambda_{0}\right)=1+\gamma\left(-\lambda_{0}\right) \gamma^{\dagger}\left(-\lambda_{0}\right)
$$

which can be obtained from the second symmetry condition in 2.3.

In addition, for $|\lambda|<\lambda_{0}$, it follows from 3.2 that

$$
\lim _{\epsilon \rightarrow 0^{+}} \delta(\lambda-i \epsilon)=\left(\mathcal{I}+\gamma(\lambda)^{\dagger} \gamma(\lambda)\right)^{-1} \lim _{\epsilon \rightarrow 0^{-}} \delta(\lambda+i \epsilon) .
$$

If we set $g(\lambda)=\left(\delta^{\dagger}(\bar{\lambda})\right)^{-1}$, we then get

$$
g_{+}(\lambda)=\left(\mathcal{I}+\gamma^{\dagger}(\lambda) \gamma(\lambda)\right) g_{-}(\lambda)
$$


Thus, we know

$$
\left(\delta^{\dagger}(\bar{\lambda})\right)^{-1}=\delta(\lambda)
$$

Similar to [6], after a direct calculation, we obtain

$$
|\delta(\lambda)| \leq \text { const }<\infty, \quad|\operatorname{det}(\delta(\lambda))| \leq \text { const }<\infty
$$

for all $\lambda$, where we define $|\mathbf{A}|=\sqrt{\left(\operatorname{tr} \mathbf{A}^{\dagger} \mathbf{A}\right)}$ for any matrix $\mathbf{A}$. Then we define

$$
\Delta(\lambda)=\left(\begin{array}{cc}
\delta(\lambda)^{-1} & 0 \\
0 & \operatorname{det}(\delta(\lambda))
\end{array}\right)
$$

Introduce

$$
M^{\Delta}(x, t ; \lambda)=M(x, t ; \lambda) \Delta(\lambda)
$$

and reverse the orientation for $|\lambda|<\lambda_{0}$ as seen in Figure 3.1 .

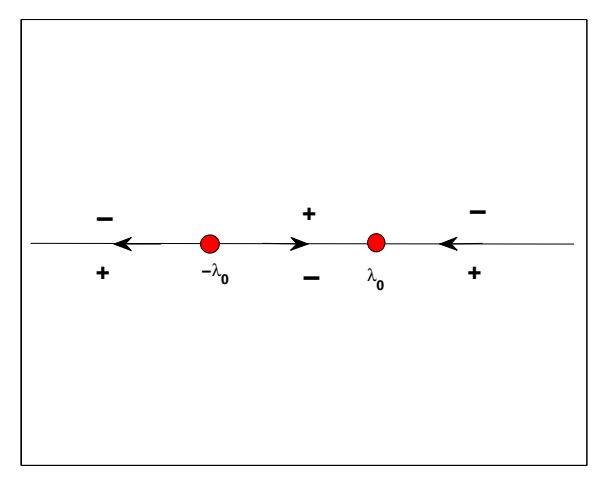

Figure 3.1: The oriented jump contour $\mathbb{R}$.

Theorem 3.1. The $M^{\Delta}$ admits the following RHP

$$
\begin{cases}M_{+}^{\Delta}(x, t ; \lambda)=M_{-}^{\Delta}(x, t ; \lambda) J^{\Delta}(x, t ; \lambda), & \lambda \in \mathbb{R} \\ M^{\Delta}(x, t ; \lambda) \rightarrow \mathcal{I}, & \lambda \rightarrow \infty\end{cases}
$$

where

$$
J^{\Delta}(\lambda)=\left(\begin{array}{cc}
\mathcal{I} & 0 \\
\frac{e^{-2 i t \theta} \rho^{\dagger}(\bar{\lambda}) \delta_{-}^{-1}(\lambda)}{\operatorname{det} \delta_{-}(\lambda)} & 1
\end{array}\right)\left(\begin{array}{cc}
\mathcal{I} & \left(\operatorname{det} \delta_{+}(\lambda)\right) e^{2 i t \theta} \delta_{+}(\lambda) \rho(\lambda) \\
0 & 1
\end{array}\right)
$$

and the vector-valued function

$$
\rho(\lambda)= \begin{cases}\frac{\gamma^{\dagger}(\bar{\lambda})}{1+\gamma(\lambda) \gamma^{\dagger}(\bar{\lambda})}, & |\lambda|<\lambda_{0} \\ -\gamma^{\dagger}(\bar{\lambda}), & |\lambda|>\lambda_{0}\end{cases}
$$




\subsection{Analytic approximations of $\rho(\lambda)$}

Our next purpose is to deform the contour, but we need to discuss the decomposition of $\rho(\lambda)$. Take

$$
L:\left\{\lambda=\lambda_{0}+\lambda_{0} \alpha e^{\frac{3 \pi i}{4}}:-\infty<\alpha \leq \sqrt{2}\right\} \cup\left\{\lambda=-\lambda_{0}+\lambda_{0} \alpha e^{\frac{\pi i}{4}}:-\infty<\alpha \leq \sqrt{2}\right\},
$$

and

$$
L_{\epsilon}:\left\{\lambda=\lambda_{0}+\lambda_{0} \alpha e^{\frac{3 \pi i}{4}}: \epsilon<\alpha \leq \sqrt{2}\right\} \cup\left\{\lambda=-\lambda_{0}+\lambda_{0} \alpha e^{\frac{\pi i}{4}}: \epsilon<\alpha \leq \sqrt{2}\right\}
$$

where $0<\epsilon \leq \sqrt{2}$.

Lemma 3.2. 6] As $0<\lambda_{0} \leq C$, there exists decomposition for the function $\rho(\lambda)$ :

$$
\rho(\lambda)=h_{1}(\lambda)+h_{2}(\lambda)+R(\lambda), \quad \lambda \in \mathbb{R},
$$

where $R(\lambda)$ is analytic in the complex plane and $h_{2}(\lambda)$ is analytically and continuously extended to L. Additionally, $R(\lambda), h_{1}(\lambda)$ and $h_{2}(\lambda)$ satisfy

$$
\begin{cases}\left|e^{2 i t \theta(\lambda)} h_{1}(\lambda)\right| \lesssim t^{-l}, & \lambda \in \mathbb{R} \\ \left|e^{2 i t \theta(\lambda)} h_{2}(\lambda)\right| \lesssim t^{-l}, & \lambda \in L \\ \left|e^{2 i t \theta(\lambda)} R(\lambda)(\lambda)\right| \lesssim e^{-16 \epsilon^{2} \lambda_{0}^{3}}, & \lambda \in L_{\epsilon}\end{cases}
$$

where positive integer $l$ is free. It follows from the Schwartz conjugate representation of (3.3) that

$$
\rho^{\dagger}(\bar{\lambda})=h_{1}^{\dagger}(\bar{\lambda})+h_{2}^{\dagger}(\bar{\lambda})+R^{\dagger}(\bar{\lambda})
$$

we obtain the similar estimates for $e^{-2 i t \theta(\lambda)} h_{1}^{\dagger}(\bar{\lambda}), e^{-2 i t \theta(\lambda)} h_{2}^{\dagger}(\bar{\lambda})$ and $e^{-2 i t \theta(\lambda)} R^{\dagger}(\bar{\lambda})$ on the contour $\mathbb{R} \cup \bar{L}$.

\subsection{Contour deformation}

We rewrite $J^{\Delta}(x, t ; \lambda)$ as $J^{\Delta}=\left(b_{-}\right)^{-1} b_{+}$, where $b_{ \pm}=\mathcal{I}+\omega_{ \pm}, \omega_{ \pm}=\omega_{ \pm}^{o}+\omega_{ \pm}^{a}$,

$$
\begin{aligned}
b_{+} & =b_{+}^{o} b_{+}^{a}=\left(\mathcal{I}+\omega_{+}^{o}\right)\left(\mathcal{I}+\omega_{+}^{a}\right) \\
& \triangleq\left(\begin{array}{cc}
\mathcal{I} & \operatorname{det} \delta(\lambda) e^{2 i \theta} \delta(\lambda) h_{1}(\lambda) \\
0 & 1
\end{array}\right)\left(\begin{array}{cc}
\mathcal{I} & \operatorname{det} \delta(\lambda) e^{2 i t \theta} \delta(\lambda)\left(h_{2}(\lambda)+R(\lambda)\right) \\
0 & 1
\end{array}\right), \\
b_{-} & =b_{-}^{o} b_{-}^{a}=\left(\mathcal{I}-\omega_{-}^{o}\right)\left(\mathcal{I}-\omega_{-}^{a}\right) \\
& \triangleq\left(\begin{array}{cc}
\mathcal{I} & 0 \\
-\frac{e^{-2 i t \theta} h_{1}^{\dagger}(\bar{\lambda}) \delta(\lambda)}{\operatorname{det} \delta(\lambda)} & 1
\end{array}\right)\left(\begin{array}{cc}
\mathcal{I} & 0 \\
-\frac{e^{-2 i t \theta}\left(h_{2}^{\dagger}(\bar{\lambda})+R^{\dagger}(\bar{\lambda})\right) \delta^{-1}(\lambda)}{\operatorname{det} \delta(\lambda)} & 1
\end{array}\right) .
\end{aligned}
$$


Lemma 3.3. Take

$$
M^{\sharp}(\lambda)= \begin{cases}M^{\Delta}(\lambda), & \lambda \in \Omega_{1} \cup \Omega_{2}, \\ M^{\Delta}(\lambda)\left(b_{-}^{a}\right)^{-1}, & \lambda \in \Omega_{3} \cup \Omega_{4} \cup \Omega_{5}, \\ M^{\Delta}(\lambda)\left(b_{+}^{a}\right)^{-1}, & \lambda \in \Omega_{6} \cup \Omega_{7} \cup \Omega_{8} .\end{cases}
$$

As a result, the function $M^{\sharp}(\lambda)$ admits the RHP on the contour $\Sigma=L \cup \bar{L} \cup \mathbb{R}$ displayed in Figure 3.2 ,

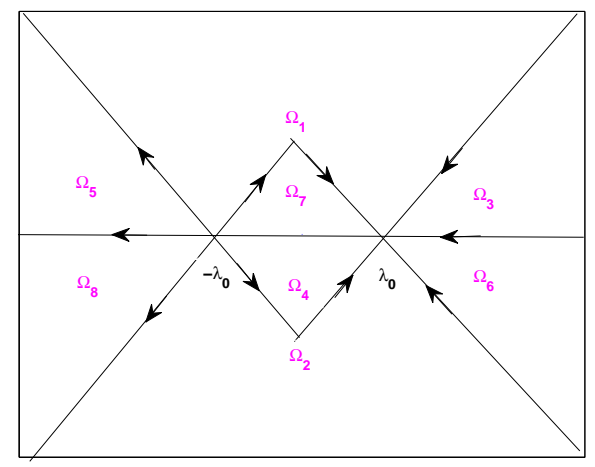

Figure 3.2: The oriented jump contour $\Sigma$.

$$
\begin{cases}M_{+}^{\sharp}(\lambda)=M_{-}^{\sharp}(\lambda) J^{\sharp}(\lambda), & \lambda \in \Sigma, \\ M^{\sharp} \rightarrow \mathcal{I}, & \lambda \rightarrow \infty,\end{cases}
$$

where

$$
J^{\sharp}=\left(b_{-}^{\sharp}\right)^{-1} b_{+}^{\sharp} \triangleq \begin{cases}\left(b_{-}^{o}\right)^{-1} b_{+}^{o}, & \lambda \in \mathbb{R}, \\ \mathcal{I}^{-1} b_{+}^{a}, & \lambda \in L, \\ \left(b_{-}^{a}\right)^{-1} \mathcal{I}, & \lambda \in \bar{L} .\end{cases}
$$

The above RHP (3.4) can be obtained (see [3]). Take

$$
\omega^{\sharp}=\omega_{-}^{\sharp}+\omega_{+}^{\sharp}, \quad \omega_{ \pm}^{\sharp}= \pm b_{ \pm}^{\sharp} \mp \mathcal{I} .
$$

In the following, the Cauchy operators $C_{ \pm}$for $\lambda \in \Sigma$ are denoted by

$$
C_{ \pm} f(\lambda)=\frac{1}{2 \pi i} \int_{\Sigma} \frac{f(\xi)}{\xi-\lambda_{ \pm}} d \xi
$$

where $f \in \mathscr{L}^{2}(\Sigma)$. Define

$$
C_{\omega^{\sharp}} f=C_{+}\left(f \omega_{-}^{\sharp}\right)+C_{-}\left(f \omega_{+}^{\sharp}\right) .
$$


Theorem 3.4. [3] Assume $\mu^{\sharp}(x, t ; \lambda) \in \mathscr{L}^{2}(\Sigma)+\mathscr{L}^{\infty}(\Sigma)$ satisfies

$$
\mu^{\sharp}=\mathcal{I}+C_{\omega} \mu^{\sharp} .
$$

Thus

$$
M^{\sharp}(\lambda)=\mathcal{I}+\frac{1}{2 \pi i} \int_{\Sigma} \frac{\mu^{\sharp}(\xi) \omega^{\sharp}(\xi)}{\xi-\lambda} d \xi
$$

represents the solution of the RHP (3.4).

Theorem 3.5. The solutions $(u(x, t), v(x, t))$ for the CSS equation 1.2 are expressed by

$$
\begin{aligned}
\mathcal{U}(x, t) & =\left(\begin{array}{l}
\mathbf{u} \\
\mathbf{v}
\end{array}\right)=2 i \lim _{\lambda \rightarrow \infty}\left(\lambda M^{\sharp}(\lambda)\right)_{12} \\
& =-\frac{1}{\pi}\left(\int_{\Sigma}\left(\mu^{\sharp}(\xi) \omega^{\sharp}(\xi) d \xi\right) d \xi\right)_{12} \\
& =-\frac{1}{\pi}\left(\left(\int_{\Sigma}\left(1-C_{\omega^{\sharp}}\right)^{-1} \mathcal{I}\right)(\xi) \omega^{\sharp}(\xi) d \xi\right)_{12} .
\end{aligned}
$$

Proof. The similar result is provided in [6].

\subsection{Contour truncation}

As seen in Figure 3.3, take $\Sigma^{\prime}=\Sigma\left(\mathbb{R} \cup L_{\epsilon} \cup \bar{L}_{\epsilon}\right)$ with the orientation. We plane to replace the RHP on $\Sigma$ with the truncated contour $\Sigma^{\prime}$ by error control.

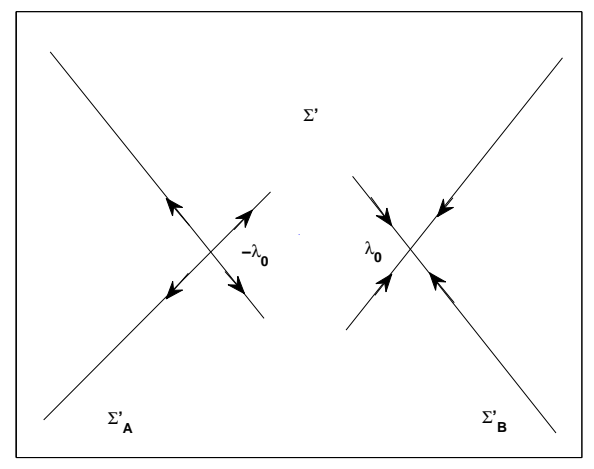

Figure 3.3: The oriented contour $\Sigma^{\prime}=\Sigma_{A}^{\prime} \cup \Sigma_{B}^{\prime}$.

Take

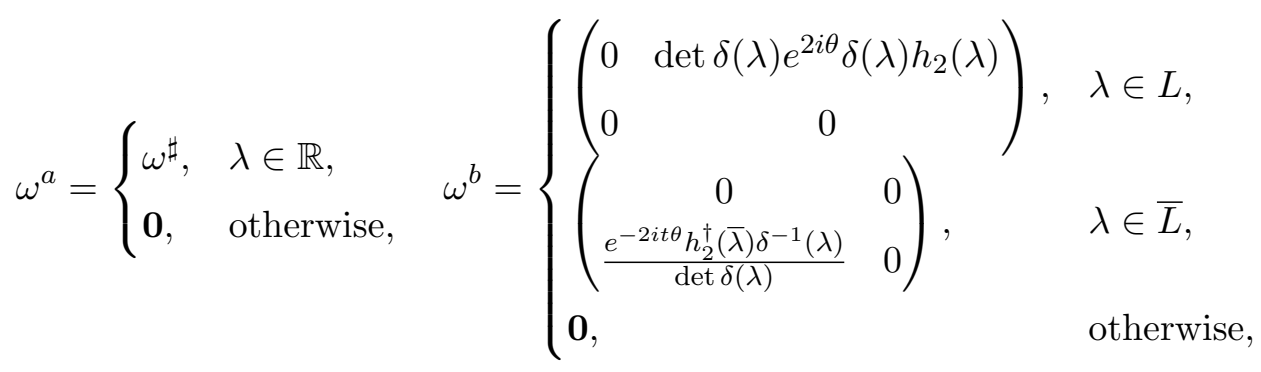




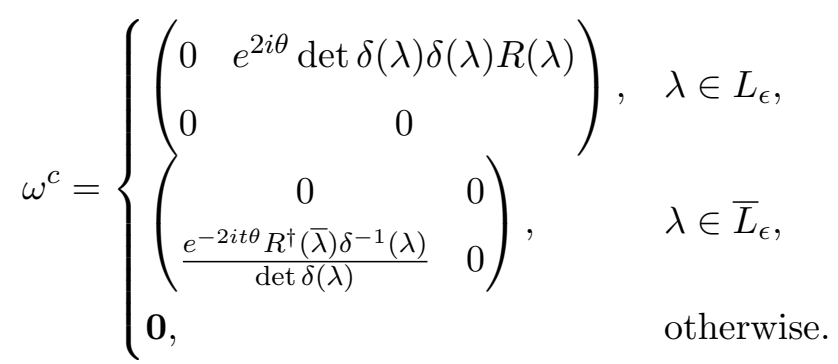

Define $\omega^{\prime}=\omega^{\sharp}-\omega^{a}-\omega^{b}-\omega^{c}$, so $\omega^{\prime}=0$ on the contour $\Sigma \backslash \Sigma^{\prime}$. Thus, $\omega^{\prime}$ is supposed on contour $\Sigma^{\prime}$ and related to $R(\lambda)$ and $R^{\dagger}(\bar{\lambda})$.

Lemma 3.6. 6] For sufficiently small $\epsilon$, as $t \rightarrow \infty$, we obtain

$$
\begin{gathered}
\left\|\omega^{a}\right\|_{\mathscr{L}^{\infty}(\mathbb{R}) \cap \mathscr{L}^{1}(\mathbb{R})} \lesssim t^{-l}, \quad\left\|\omega^{b}\right\|_{\mathscr{L}^{\infty}(L \cup \bar{L}) \cap \mathscr{L}^{1}(L \cup \bar{L})} \lesssim t^{-l}, \\
\left\|\omega^{c}\right\|_{\mathscr{L}^{\infty}\left(L_{\epsilon} \cup \bar{L}_{\epsilon}\right) \cap \mathscr{L}^{1}\left(L_{\epsilon} \cup \bar{L}_{\epsilon}\right)} \lesssim e^{-16 \epsilon^{2} \tau}, \quad\left\|\omega^{\prime}\right\|_{\mathscr{L}^{2}(\Sigma)} \lesssim \tau^{-1 / 4}, \quad\left\|\omega^{\prime}\right\|_{\mathscr{L}^{1}(\Sigma)} \lesssim \tau^{-1 / 2},
\end{gathered}
$$

where $\tau=\lambda_{0}^{3} t$.

Lemma 3.7. [6] In the case $0<\lambda_{0} \leq C$, as $\tau \rightarrow \infty$, the inverse $\left(1-C_{\omega^{\prime}}\right)^{-1}: \mathscr{L}^{2}(\Sigma) \rightarrow$ $\mathscr{L}^{2}(\Sigma)$ exists, and has uniform boundedness

$$
\left\|\left(1-C_{\omega^{\prime}}\right)^{-1}\right\|_{\mathscr{L}^{2}(\Sigma)} \lesssim 1
$$

Besides

$$
\left\|\left(1-C_{\omega^{\sharp}}\right)^{-1}\right\|_{\mathscr{L}^{2}(\Sigma)} \lesssim 1 .
$$

Lemma 3.8. The integral equation has estimate as $\tau \rightarrow \infty$

$$
\int_{\Sigma}\left(\left(1-C_{\omega^{\sharp}}\right)^{-1} \mathcal{I}\right)(\xi) \omega^{\sharp}(\xi) d \xi=\int_{\Sigma}\left(\left(1-C_{\omega^{\prime}}\right)^{-1} \mathcal{I}\right)(\xi) \omega^{\prime}(\xi) d \xi+O\left(\frac{1}{\tau^{l}}\right) .
$$

Proof. After a simple calculation, we find

$$
\begin{aligned}
\left(\left(1-\omega^{\sharp}\right)^{-1} \mathcal{I}\right) \omega^{\sharp}= & \left(\left(1-C \omega^{\prime}\right)^{-1} \mathcal{I}\right) \omega^{\prime}+\omega^{e}+\left(\left(1-\omega^{\prime}\right)^{-1}\left(C_{\omega^{e}} \mathcal{I}\right)\right) \omega^{\sharp} \\
& +\left(\left(1-\omega^{\prime}\right)^{-1}\left(C_{\omega^{\prime}} \mathcal{I}\right)\right) \omega^{e}+\left(\left(1-C_{\omega^{\prime}}\right)^{-1} C_{\omega^{e}}\left(1-C_{\omega^{\sharp}}\right)^{-1}\right)\left(C_{\omega^{\sharp}} \mathcal{I}\right) \omega^{\sharp} .
\end{aligned}
$$

Then from Lemma 3.6, we have

$$
\begin{aligned}
&\left\|\omega^{e}\right\|_{\mathscr{L}^{1}(\Sigma)} \leq\left\|\omega^{a}\right\|_{\mathscr{L}^{1}(\mathbb{R})}+\left\|\omega^{b}\right\|_{\mathscr{L}^{1}\left(L_{\epsilon} \cup \bar{L}_{\epsilon}\right)} \lesssim \tau^{-l}, \\
&\left\|\left(\left(1-C_{\omega^{\prime}}\right)^{-1}\left(C_{\omega^{e}} \mathcal{I}\right)\right) \omega^{\sharp}\right\|_{\mathscr{L}^{1}(\Sigma)} \leq\left\|\left(1-C_{\omega^{\prime}}\right)^{-1}\right\|_{\mathscr{L}^{2}(\Sigma)}\left\|C_{\omega^{e}} \mathcal{I}\right\|_{\mathscr{L}^{2}(\Sigma)}\left\|\omega^{\sharp}\right\|_{\mathscr{L}^{2}(\Sigma)} \\
& \leq\left\|\omega^{e}\right\|_{\mathscr{L}^{2}(\Sigma)}\left\|\omega^{\sharp}\right\|_{\mathscr{L}^{2}(\Sigma)} \lesssim t^{-l-1 / 4}, \\
&\left\|\left(\left(1-C_{\omega^{\prime}}\right)^{-1}\left(C_{\omega^{\prime}} \mathcal{I}\right)\right) \omega^{e}\right\|_{\mathscr{L}^{1}(\Sigma)} \leq\left\|\left(1-C_{\omega^{\prime}}\right)^{-1}\right\|_{\mathscr{L}^{2}(\Sigma)}\left\|C_{\omega^{\prime}} \mathcal{I}\right\|_{\mathscr{L}^{2}(\Sigma)}\left\|\omega^{e}\right\|_{\mathscr{L}^{2}(\Sigma)} \\
& \leq\left\|\omega^{\prime}\right\|_{\mathscr{L}^{2}(\Sigma)}\left\|\omega^{e}\right\|_{\mathscr{L}^{2}(\Sigma)} \lesssim t^{-l-1 / 4},
\end{aligned}
$$




$$
\begin{aligned}
& \left\|\left(\left(1-C_{\omega^{\prime}}\right)^{-1} C_{\omega^{e}}\left(1-C_{\omega^{\sharp}}\right)^{-1}\right)\left(C_{\omega^{\sharp}} \mathcal{I}\right) \omega^{\sharp}\right\|_{\mathscr{L}^{1}(\Sigma)} \\
= & \left\|\left(1-C_{\omega^{\prime}}\right)^{-1}\right\|_{\mathscr{L}^{2}(\Sigma)}\left\|C_{\omega^{e}}\right\|_{\mathscr{L}^{2}(\Sigma)}\left\|\left(1-C_{\omega^{\sharp}}\right)^{-1}\right\|_{\mathscr{L}^{2}(\Sigma)}\left\|C_{\omega^{\sharp}} \mathcal{I}\right\|_{\mathscr{L}^{2}(\Sigma)}\left\|\omega^{\sharp}\right\|_{\mathscr{L}^{2}(\Sigma)} \\
\lesssim & \left\|\omega^{e}\right\|_{\mathscr{L}^{\infty}(\Sigma)}\left\|\omega^{\sharp}\right\|_{\mathscr{L}^{2}(\Sigma)}^{2} \lesssim t^{-l-1 / 2} .
\end{aligned}
$$

This finishes proof of Lemma 3.8 .

Lemma 3.9. The solution admits the following asymptotics, as $\tau \rightarrow \infty$

$$
\mathcal{U}(x, t)=\left(\begin{array}{l}
\mathbf{u}(x, t) \\
\mathbf{v}(x, t)
\end{array}\right)=-\frac{1}{\pi}\left(\int_{\Sigma^{\prime}}\left(\left(1-C_{\omega^{\prime}}\right)^{-1} \mathcal{I}\right)(x, t ; \xi) \omega^{\prime}(x, t, \xi) d \xi\right)_{12}+O\left(\frac{1}{\tau^{l}}\right)
$$

Proof. The lemma follows by Theorem 3.5 and Lemma 3.8 .

Here take $L^{\prime}=L \backslash L_{\epsilon}$ and $\Sigma^{\prime}=L^{\prime} \cup \bar{L}^{\prime}$. Let $\mu^{\prime}=\left(1-C_{\omega^{\prime}}\right)^{-1} \mathcal{I}$. Then

$$
M^{\prime}(\lambda)=\mathcal{I}+\frac{1}{2 \pi i} \int_{\Sigma^{\prime}} \frac{\mu^{\prime}(\xi) \omega^{\prime}(\xi)}{\xi-\lambda} d \xi
$$

meets

$$
\begin{cases}M_{+}^{\prime}(\lambda)=M_{-}^{\prime}(\lambda) J^{\prime}(\lambda), & \lambda \in \Sigma^{\prime} \\ M^{\prime}(\lambda) \rightarrow \mathcal{I}, & \lambda \rightarrow \infty\end{cases}
$$

where

$$
\begin{aligned}
J^{\prime} & =b_{-}^{-1} b_{+}^{\prime}, \quad b_{-}^{\prime}=\mathcal{I}, \quad \lambda \in L^{\prime}, \\
b_{+}^{\prime} & =\left(\begin{array}{cc}
\mathcal{I} & 0 \\
e^{2 i t \theta(\lambda)} \operatorname{det}(\delta(\lambda)) R(\lambda) & 1
\end{array}\right), \\
b_{+}^{\prime}=\mathcal{I}, \quad b_{-}^{\prime} & =\left(\begin{array}{cc}
\mathcal{I} & -\frac{e^{-2 i t \theta(\lambda)} \delta^{-1}(\lambda) R^{\dagger}(\bar{\lambda})}{\operatorname{det} \delta(\lambda)} \\
0 & 1
\end{array}\right), \quad \lambda \in \bar{L}^{\prime} .
\end{aligned}
$$

3.5. Noninteraction of disconnected contour

Choose $\omega^{\prime}=\omega_{+}^{\prime}+\omega_{-}^{\prime}$, where $\omega_{ \pm}^{\prime}= \pm b_{ \pm}^{\prime}-\mp \mathcal{I}$. Let the contour $\Sigma^{\prime}=\Sigma_{\mathbf{A}}^{\prime} \cup \Sigma_{\mathbf{B}}^{\prime}$ and $\omega_{ \pm}^{\prime}=\omega_{\mathbf{A}_{ \pm}}^{\prime}+\omega_{\mathbf{B}_{ \pm}}^{\prime}$, where $\omega_{\mathbf{A}_{ \pm}}^{\prime}(\lambda)=0$ for $\lambda \in \Sigma_{\mathbf{B}^{\prime}}^{\prime}, \omega_{\mathbf{B}_{ \pm}}^{\prime}(\lambda), \omega_{\mathbf{B}_{ \pm}}^{\prime}(\lambda)=0$ for $\lambda \in \Sigma_{\mathbf{A}^{\prime}}^{\prime}$. Give the operators $C_{\omega_{\mathbf{A}}^{\prime}}$ and $C_{\omega_{\mathbf{B}}^{\prime}}: \mathscr{J}^{\infty}\left(\Sigma^{\prime}\right)+\mathscr{J}^{2}\left(\Sigma^{\prime}\right) \rightarrow \mathscr{J}^{2}\left(\Sigma^{\prime}\right)$ as in 3.5 .

Lemma 3.10. 6

$$
\begin{gathered}
\left\|C_{\omega_{\mathbf{B}}^{\prime} C_{\omega_{\mathbf{A}}^{\prime}}}\right\|=\left\|C_{\omega_{\mathbf{A}}^{\prime} C_{\omega_{\mathbf{B}}^{\prime}}}\right\|_{\mathscr{J}^{2}\left(\Sigma^{\prime}\right)} \lesssim \lambda_{0} \tau^{-1 / 2}, \\
\left\|C_{\omega_{\mathbf{B}}^{\prime}} C_{\omega_{\mathbf{A}}^{\prime}}\right\| \mathscr{J} \infty\left(\Sigma^{\prime}\right) \rightarrow \mathscr{J}^{2}\left(\Sigma^{\prime}\right),\left\|C_{\omega_{\mathbf{A}}^{\prime}} C_{\omega_{\mathbf{B}}^{\prime}}\right\|_{\mathscr{J} \infty\left(\Sigma^{\prime}\right) \rightarrow \mathscr{J}^{2}\left(\Sigma^{\prime}\right)} \leq \lambda_{0} \tau^{-3 / 4} .
\end{gathered}
$$

Proof. Together with Lemmas 3.6 and 3.7 , we obtain (3.6). 
Lemma 3.11. As $\tau \rightarrow \infty$,

$$
\begin{aligned}
\int_{\Sigma^{\prime}}\left(\left(1-C_{\omega^{\prime}}\right)^{-1} \mathcal{I}\right)(\xi) \omega^{\prime}(\xi) d \xi= & \int_{\Sigma_{\mathbf{A}}^{\prime}}\left(\left(1-C_{\omega_{\mathbf{A}}^{\prime}}\right)^{-1} \mathcal{I}\right)(\xi) \omega^{\prime}(\xi) d \xi \\
& +\int_{\Sigma_{\mathbf{B}}^{\prime}}\left(\left(1-C_{\omega_{\mathbf{A}}^{\prime}}\right)^{-1} \mathcal{I}\right)(\xi) \omega^{\prime}(\xi) d \xi+O\left(\frac{1}{\tau}\right)
\end{aligned}
$$

Proof. From the following relation

$$
\begin{aligned}
& \left(1-C_{\omega_{\mathbf{A}}^{\prime}}-C_{\omega_{\mathbf{B}}^{\prime}}\right)\left(1+C_{\omega_{\mathbf{A}}^{\prime}}\left(1-C_{\omega_{\mathbf{A}}^{\prime}}\right)^{-1}+C_{\omega_{\mathbf{B}}^{\prime}}\left(1-C_{\omega_{\mathbf{B}}^{\prime}}\right)^{-1}\right) \\
= & 1-C_{\omega_{\mathbf{B}}^{\prime}} C_{\omega_{\mathbf{A}}^{\prime}}\left(1-C_{\omega_{\mathbf{A}}^{\prime}}\right)^{-1}-C_{\omega_{\mathbf{A}}^{\prime}} C_{\omega_{\mathbf{B}}^{\prime}}\left(1-C_{\omega_{\mathbf{B}}^{\prime}}\right)^{-1},
\end{aligned}
$$

we find

$$
\begin{aligned}
\left(1-C_{\omega^{\prime}}\right)^{-1}=\left(1+C_{\omega^{\prime}}\right)^{-1}=1 & +C_{\omega_{\mathbf{A}}^{\prime}}\left(1-C_{\omega_{\mathbf{A}}^{\prime}}\right)^{-1}+C_{\omega_{\mathbf{B}}^{\prime}}\left(1-C_{\omega_{\mathbf{B}}^{\prime}}\right)^{-1} \\
+ & \left(1+C_{\omega_{\mathbf{A}}^{\prime}}\left(1-C_{\omega_{\mathbf{A}}^{\prime}}\right)^{-1}+C_{\omega_{\mathbf{B}}^{\prime}}\left(1-C_{\omega_{\mathbf{B}}^{\prime}}\right)^{-1}\right) \\
& \times\left(1-C_{\omega_{\mathbf{B}}^{\prime}} C_{\omega_{\mathbf{A}}^{\prime}}\left(1-C_{\omega_{\mathbf{A}}^{\prime}}\right)^{-1}-C_{\omega_{\mathbf{A}}^{\prime}} C_{\omega_{\mathbf{B}}^{\prime}}\left(1-C_{\omega_{\mathbf{B}}^{\prime}}\right)^{-1}\right)^{-1} \\
& \times\left(C_{\omega_{\mathbf{B}}^{\prime}} C_{\omega_{\mathbf{A}}^{\prime}}\left(1-C_{\omega_{\mathbf{A}}^{\prime}}\right)^{-1}+C_{\omega_{\mathbf{A}}^{\prime}} C_{\omega_{\mathbf{B}}^{\prime}}\left(1-C_{\omega_{\mathbf{B}}^{\prime}}\right)^{-1}\right) .
\end{aligned}
$$

Together with Lemmas 3.6, 3.7 and 3.10, we obtain Lemma 3.11.

Note. We also write the restriction $C_{\omega_{\mathbf{A}}^{\prime}} \mid \mathscr{J}^{2}\left(\Sigma_{\mathbf{A}}^{\prime}\right)$ as $C_{\omega_{\mathbf{B}}^{\prime}}$.

Lemma 3.12. As $\tau \rightarrow \infty$, we have

$$
\begin{aligned}
\mathcal{U}(x, t)=\left(\begin{array}{l}
\mathbf{u} \\
\mathbf{v}
\end{array}\right)= & -\frac{1}{\pi}\left(\int_{\Sigma_{\mathbf{A}}^{\prime}}\left(\left(1-C_{\omega_{\mathbf{A}}^{\prime}}\right)^{-1} \mathcal{I}\right)(x, t ; \xi) \omega_{\mathbf{A}}^{\prime}(x, t ; \xi) d \xi\right)_{12} \\
& -\frac{1}{\pi}\left(\int_{\Sigma_{\mathbf{B}}^{\prime}}\left(\left(1-C_{\omega_{\mathbf{B}}^{\prime}}\right)^{-1} \mathcal{I}\right)(x, t ; \xi) \omega_{\mathbf{B}}^{\prime}(x, t ; \xi) d \xi\right)_{12}+O\left(\frac{1}{\tau}\right) .
\end{aligned}
$$

\subsection{The model Riemann-Hilbert problem}

Extend $\Sigma_{\mathbf{A}}^{\prime}$ and $\Sigma_{\mathbf{B}}^{\prime}$ to the following contours

$$
\widehat{\Sigma}_{\mathbf{A}}^{\prime}=\left\{\lambda=-\lambda_{0}+\lambda_{0} \alpha e^{\frac{ \pm i \pi}{4}}: \alpha \in \mathbb{R}\right\}, \quad \widehat{\Sigma}_{\mathbf{B}}^{\prime}=\left\{\lambda=-\lambda_{0}+\lambda_{0} \alpha e^{\frac{ \pm 3 i \pi}{4}}: \alpha \in \mathbb{R}\right\}
$$

respectively, and give $\widehat{\omega}_{\mathbf{A}}^{\prime}, \widehat{\omega}_{\mathbf{B}}^{\prime}$ on $\widehat{\Sigma}_{\mathbf{A}}^{\prime}, \widehat{\Sigma}_{\mathbf{B}}^{\prime}$ as

$$
\widehat{\omega}_{\mathbf{A}_{ \pm}}^{\prime}=\left\{\begin{array}{ll}
\omega_{A_{ \pm}}^{\prime}(\lambda), & \lambda \in \Sigma_{\mathbf{A}}^{\prime}, \\
0, & \lambda \in \widehat{\Sigma}_{\mathbf{A}}^{\prime} \backslash \Sigma_{\mathbf{A}}^{\prime},
\end{array} \quad \widehat{\omega}_{\mathbf{B}_{ \pm}}^{\prime}= \begin{cases}\omega_{\mathbf{B}_{ \pm}}^{\prime}(\lambda), & \lambda \in \Sigma_{\mathbf{B}}^{\prime}, \\
0, & \lambda \in \widehat{\Sigma}_{\mathbf{B}}^{\prime} \backslash \Sigma_{\mathbf{B}}^{\prime}\end{cases}\right.
$$


Let $\Sigma_{\mathbf{A}}$ and $\Sigma_{\mathbf{B}}$ denote the contours $\left\{\lambda=\lambda_{0} \alpha e^{ \pm \frac{i \pi}{4}}: \alpha \in \mathbb{R}\right\}$ shown in Figure 3.4. The scaling operators $N_{\mathbf{A}}$ and $N_{\mathbf{B}}$ is given by

$$
\begin{aligned}
& N_{\mathbf{A}}: \mathscr{J}^{2}\left(\widehat{\Sigma}_{\mathbf{A}}^{\prime}\right) \rightarrow \mathscr{J}^{2}\left(\Sigma^{\mathbf{A}}\right), \quad f(\lambda) \mapsto\left(N_{\mathbf{A}} f\right)(\lambda)=f\left(\frac{\lambda}{4 \sqrt{3 \lambda_{0} t}}-\lambda_{0}\right) \\
& N_{\mathbf{B}}: \mathscr{J}^{2}\left(\widehat{\Sigma}_{\mathbf{B}}^{\prime}\right) \rightarrow \mathscr{J}^{2}\left(\Sigma^{\mathbf{B}}\right), \quad f(\lambda) \mapsto\left(N_{\mathbf{B}} f\right)(\lambda)=f\left(\frac{\lambda}{4 \sqrt{3 \lambda_{0} t}}+\lambda_{0}\right),
\end{aligned}
$$

and take

$$
\omega_{\mathbf{A}}=N_{\mathbf{A}} \widehat{\omega}_{\mathbf{A}}^{\prime}, \quad \omega_{\mathbf{B}}=N_{\mathbf{B}} \widehat{\omega}_{\mathbf{B}}^{\prime}
$$

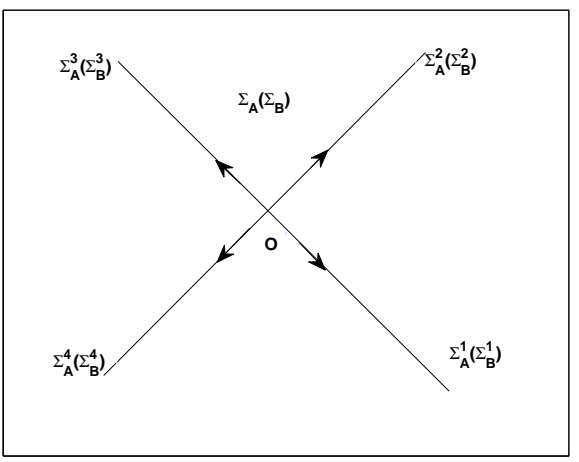

Figure 3.4: The oriented contour $\Sigma_{A}$ or $\Sigma_{B}$.

A simple replacement yields

$$
C_{\widehat{\omega}_{\mathbf{A}}^{\prime}}=N_{\mathbf{A}}^{-1} C_{\omega_{\mathbf{A}}} N_{\mathbf{A}}, \quad C_{\widehat{\omega}_{\mathbf{B}}^{\prime}}=N_{\mathbf{B}}^{-1} C_{\omega_{\mathbf{A}}} N_{\mathbf{B}}
$$

where $C_{\omega_{\mathbf{A}}}: \mathscr{J}^{2}\left(\Sigma_{\mathbf{A}}\right) \rightarrow \mathscr{J}^{2}\left(\Sigma_{\mathbf{A}}\right)$ is bounded, similar to $C_{\omega_{\mathbf{B}}}$.

On the other hand, we infer that

$$
\omega_{\mathbf{A}}=\omega_{\mathbf{A}+}=\left(\begin{array}{cc}
0 & \left(N_{\mathbf{A}} s_{1}\right)(\lambda) \\
0 & 0
\end{array}\right)
$$

on

$$
L_{\mathbf{A}}=\left\{\lambda=\alpha \lambda_{0} 4 \sqrt{3 \lambda_{0} t} e^{-\frac{3 \pi i}{4}}:-\epsilon<\alpha<+\infty\right\},
$$

and

$$
\omega_{\mathbf{A}}=\omega_{\mathbf{A}-}=\left(\begin{array}{cc}
0 & 0 \\
\left(N_{\mathbf{A}} s_{2}\right)(\lambda) & 0
\end{array}\right)
$$

on $\bar{L}_{\mathbf{A}}$, where

$$
s_{1}(\lambda)=\operatorname{det} \delta(\lambda) e^{2 i \theta(\lambda) t} \delta(\lambda) R(\lambda), \quad s_{2}(\lambda)=\frac{e^{-2 i \theta(\lambda) t} R^{\dagger}(\bar{\lambda}) \delta(\lambda)^{-1}}{\operatorname{det} \delta(\lambda)} .
$$


Lemma 3.13. As $\lambda \in L_{\mathbf{A}}$ and $t \rightarrow \infty$, we have

$$
\left|\left(N_{\mathbf{A}}\right) \widetilde{\delta}(\lambda)\right| \lesssim t^{-l}
$$

where

$$
\widetilde{\delta}(\lambda)=e^{2 i \theta(\lambda) t}[\delta(\lambda)-\operatorname{det} \delta(\lambda) \mathcal{I}] R(\lambda) .
$$

Proof. It follows from (3.2), 3.1 and 3.8 that

$$
\begin{cases}\widetilde{\delta}_{+}(\lambda)=e^{2 i t \theta} f(\lambda)+\widetilde{\delta}_{-}(\lambda)\left(1+|\gamma(\lambda)|^{2}\right), & |\lambda|<\lambda_{0}, \\ \widetilde{\delta}(\lambda) \rightarrow 0, & \lambda \rightarrow \infty\end{cases}
$$

where

$$
f(\lambda)=\delta_{-}\left(\gamma^{\dagger} \gamma R-|\gamma|^{2} R\right)(\lambda)
$$

By using Plemelj formula, the solution $\widetilde{\delta}(\lambda)$ reaches to

$$
\widetilde{\delta}(\lambda)=X(\lambda) \int_{-\lambda_{0}}^{\lambda_{0}} \frac{e^{2 i t \theta(\xi) f(\xi)}}{X_{+}(\xi)(\xi-\lambda)} d \xi, \quad X(\lambda)=e^{\frac{1}{2 \pi i} \int_{-\lambda_{0}}^{\lambda_{0}} \frac{1+|\gamma(\xi)|^{2}}{\xi-\lambda} d \xi}
$$

From

$$
\gamma^{\dagger} \gamma R-|\gamma|^{2} R=\gamma^{\dagger} \gamma(R-\rho)-|\gamma|^{2}(R-\rho),
$$

we obtain $f(\lambda)=O\left(\left(\lambda^{2}-\lambda_{0}^{2}\right)^{l}\right)$. Then we decompose $f(\lambda)$ two parts: $f(\lambda)=f_{1}(\lambda)+f_{2}(\lambda)$, where $f_{2}(\lambda)$ is analytically and continuously extended to $L_{t}$ and meets

$$
\begin{cases}\left|e^{2 i t \theta(\lambda)} f_{1}(\lambda)\right| \lesssim \frac{1}{\left(1+|\lambda|^{2}\right) t}, & \lambda \in \mathbb{R} \\ \left|e^{2 i t \theta(\lambda)} f_{1}(\lambda)\right| \lesssim \frac{1}{\left(1+|\lambda|^{2}\right) t^{l}}, & \lambda \in L_{t}\end{cases}
$$

where

$$
\begin{aligned}
L_{t}: \lambda= & \left\{\lambda_{0} \alpha e^{3 \pi i / 4}: 0 \leq \alpha \leq \sqrt{2}-\frac{1}{\sqrt{2} t}\right\} \\
& \cup\left\{\lambda=\lambda_{0} / t-\lambda_{0}+\lambda_{0} \alpha e^{\pi i / 4}: 0 \leq \alpha \leq \sqrt{2}-\frac{1}{\sqrt{2} t}\right\}
\end{aligned}
$$

is shown in Figure 3.5 .

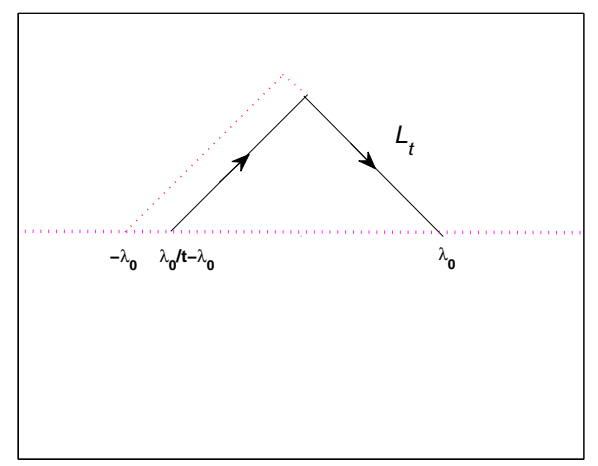

Figure 3.5: The contour $L_{t}$. 
When $\lambda \in L_{\mathbf{A}}$, we have

$$
\left(N_{A} \widetilde{\delta}\right)(\lambda)=\mathcal{I}_{1}+\mathcal{I}_{2}+\mathcal{I}_{3}
$$

with

$$
\begin{aligned}
& \left|\mathcal{I}_{1}\right| \lesssim \int_{-\lambda_{0}}^{\lambda_{0} / t-\lambda_{0}} \frac{|f(\xi)|}{\xi+\lambda_{0}-\lambda / 4 \sqrt{3 t \lambda_{0}}} d \xi \leq t^{-l} \log \left|1-\frac{4 \lambda_{0} \sqrt{3 \lambda_{0}}}{\lambda \sqrt{t}}\right| \lesssim t^{-l-1 / 2}, \\
& \left|\mathcal{I}_{2}\right| \lesssim \int_{\lambda_{0} / t-\lambda_{0}}^{\lambda_{0}} \frac{\left|e^{2 i \theta} f_{1}(\xi)\right|}{\xi+\lambda_{0}-\lambda / 4 \sqrt{3 t \lambda_{0}}} d \xi \leq t^{-l} \frac{\sqrt{2} t}{\lambda_{0}}\left(2 \lambda_{0}-\lambda_{0} / t\right) \lesssim t^{-l+1},
\end{aligned}
$$

with the help of Cauchy's Theorem, the original integral interval $\left(\lambda_{0} / t-\lambda_{0}, \lambda_{0}\right)$ in $\mathcal{I}_{3}$ can be replaced by contour $L_{t}$. Following the similar way, $\left|\mathcal{I}_{3}\right| \lesssim t^{-l+1}$, we can easily obtain Lemma 3.13 .

Similarly, we have

$$
\left|\left(N_{\mathbf{A}} \widehat{\delta}\right)(\lambda)\right| \lesssim t^{-l}, \quad \lambda \in \bar{L}_{\mathbf{A}}, \quad t \rightarrow \infty
$$

where

$$
\widehat{\delta}(\lambda)=e^{-2 i \theta(\lambda) t} R^{\dagger}(\bar{\lambda})\left[\delta(\lambda)^{-1}-\left[\operatorname{det} \delta^{-1}(\lambda)\right] \mathcal{I}\right] .
$$

Take $J^{\mathbf{A}^{0}}=\left(\mathcal{I}-\omega_{\mathbf{A}^{0}-}\right)^{-1}\left(\mathcal{I}-\omega_{\mathbf{A}^{0}+}\right)$, where

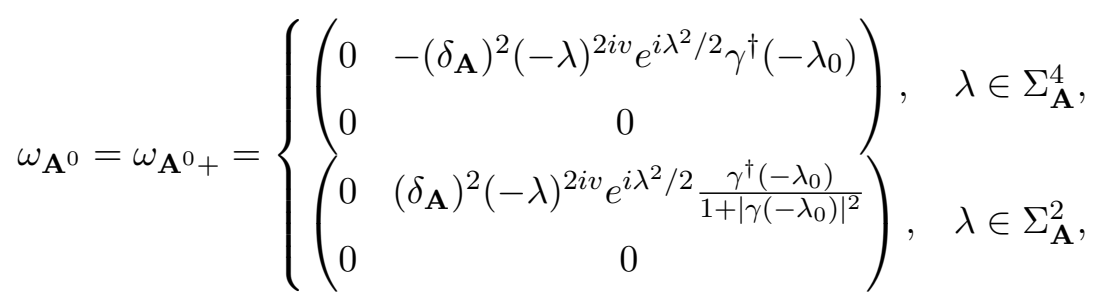

and

$$
\delta_{\mathbf{A}}=e^{\chi\left(-\lambda_{0}\right)-8 i \tau}(192 \tau)^{-i v / 2}
$$

with

$$
\omega_{\mathbf{A}^{0}}=\omega_{\mathbf{A}^{0}-}=\left\{\begin{array}{cc}
0 & 0 \\
-\left(\delta_{\mathbf{A}}\right)^{-2}(-\lambda)^{-2 i v} e^{-i \lambda^{2} / 2} \gamma\left(-\lambda_{0}\right) & 0 \\
0 & 0 \\
\left(\delta_{\mathbf{A}}\right)^{2}(-\lambda)^{-2 i v} e^{-i \lambda^{2} / 2 \frac{\gamma\left(-\lambda_{0}\right)}{1+\left|\gamma\left(-\lambda_{0}\right)\right|^{2}}} & 0
\end{array}\right), \quad \lambda \in \Sigma_{\mathbf{A}}^{3},
$$

It follows from $(3.7)$ and $[6]$ that

$$
\left\|\omega_{\mathbf{A}}-\omega_{\mathbf{A}^{0}}\right\|_{\mathscr{J}^{\infty}}\left(\Sigma_{\mathbf{A}}\right) \cap \mathscr{J}^{1}\left(\Sigma_{\mathbf{A}}\right) \cap \mathscr{J}^{2}\left(\Sigma_{\mathbf{A}}\right) \lesssim \lambda_{0} t^{1 / 2} \log (t) .
$$


Therefore

$$
\begin{aligned}
& \int_{\Sigma_{\mathbf{A}}^{\prime}}\left(\left(1-C_{\omega_{\mathbf{A}}^{\prime}}\right)^{-1} \mathcal{I}\right)(\xi) \omega_{\mathbf{A}}^{\prime}(\xi) d \xi \\
= & \int_{\widehat{\Sigma}_{\mathbf{A}}}\left(\left(1-C_{\widehat{\omega}_{\mathbf{A}}^{\prime}}\right)^{-1} \mathcal{I}\right)(\xi) \widehat{\omega}_{\mathbf{A}}^{\prime}(\xi) d \xi \\
= & \int_{\widehat{\Sigma}_{\mathbf{A}}^{\prime}}\left(N_{\mathbf{A}}^{-1}\left(1-C_{\omega_{\mathbf{A}}}\right)^{-1} N_{\mathbf{A}} \mathcal{I}\right)(\xi) \widehat{\omega}_{\mathbf{A}}^{\prime}(\xi) d \xi \\
= & \int_{\widehat{\Sigma}_{\mathbf{A}}^{\prime}}\left(\left(1-C_{\omega_{\mathbf{A}}}\right)^{-1} \mathcal{I}\right)\left(\xi+\lambda_{0}\right) 4 \sqrt{3 t \lambda_{0}} N_{\mathbf{A}} \widehat{\omega}_{\mathbf{A}}^{\prime}\left(\left(\xi+\lambda_{0}\right) 4 \sqrt{3 t \lambda_{0}}\right) d \xi \\
= & \frac{1}{4 \sqrt{3 t \lambda_{0}}} \int_{\Sigma_{\mathbf{A}}}\left(\left(1-C_{\omega_{\mathbf{A}}}\right)^{-1} \mathcal{I}\right)(\xi) \omega_{\mathbf{A}}(\xi) \\
= & \frac{1}{4 \sqrt{3 t \lambda_{0}}} \int_{\Sigma_{\mathbf{A}}}\left(\left(1-C_{\omega_{\mathbf{A}^{0}}}\right)^{-1} \mathcal{I}\right)(\xi) \omega_{\mathbf{A}}(\xi)+O\left(\frac{\log t}{t}\right) .
\end{aligned}
$$

Together with a similar computation for $\mathbf{B}$ yields

$$
\begin{aligned}
\mathcal{U}(x, t)=\left(\begin{array}{c}
\mathbf{u}(x, t) \\
\mathbf{v}(x, t)
\end{array}\right)= & -\frac{1}{\pi} \frac{1}{4 \sqrt{3 \lambda_{0} t}}\left(\int_{\Sigma_{\mathbf{A}}}\left(\left(1-C_{\omega_{\mathbf{A}^{0}}}\right)^{-1} \mathcal{I}\right)(\xi) \omega_{\mathbf{A}^{0}}(\xi) d \xi\right)_{12} \\
& -\frac{1}{\pi} \frac{1}{4 \sqrt{3 \lambda_{0} t}}\left(\int_{\Sigma_{\mathbf{B}}}\left(\left(1-C_{\omega_{\mathbf{B}^{0}}}\right)^{-1} \mathcal{I}\right)(\xi) \omega_{\mathbf{B}^{0}}(\xi) d \xi\right)_{12}+O\left(\frac{\log t}{t}\right) .
\end{aligned}
$$

For $\lambda \in \mathbb{C} \backslash \Sigma_{A}$, let

$$
M^{A^{0}}(\lambda)=\mathcal{I}+\frac{1}{2 \pi i} \int_{\Sigma_{A}} \frac{\left(\left(1-C_{\omega_{A^{0}}}\right)^{-1}\right)(\xi) \omega_{A^{0}}(\xi)}{\xi-\lambda} d \xi
$$

Then $M^{\mathbf{A}^{0}}$ admits

$$
\begin{cases}M_{+}^{\mathbf{A}^{0}}(\lambda)=M_{-}^{\mathbf{A}^{0}}(\lambda) J^{\mathbf{A}^{0}}(\lambda), & \lambda \in \Sigma_{\mathbf{A}} \\ M^{\mathbf{A}^{0}}(\lambda) \rightarrow \mathcal{I}, & \lambda \rightarrow \infty\end{cases}
$$

Particularly

$$
M^{\mathbf{A}^{0}}(\lambda)=\mathcal{I}+\frac{M_{1}^{\mathbf{A}^{0}}}{\lambda}+O\left(\frac{1}{\lambda^{2}}\right), \quad \lambda \rightarrow \infty,
$$

then

$$
M_{1}^{\mathbf{A}^{0}}=-\frac{1}{2 \pi i} \int_{\Sigma_{\mathbf{A}}}\left(\left(1-C_{\omega_{\mathbf{A}^{0}}}\right)^{-1} \mathcal{I}\right)(\xi) \omega_{\mathbf{A}^{0}}(\xi) d \xi .
$$

A similar RHP for $\mathbf{B}^{0}$ on $\Sigma_{\mathbf{B}}$ reads

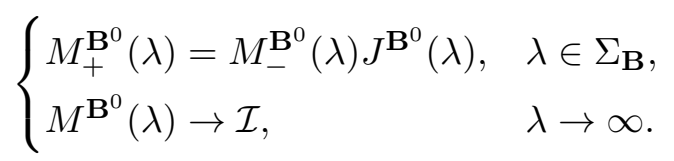

Utilizing $3.9-3.11$ and $\omega_{\mathbf{B}^{0}}$, one has

$$
J^{\mathbf{A}^{0}}(\lambda)=\bar{\tau}\left(J^{\mathbf{B}^{0}}\right)(-\bar{\lambda}) \tau .
$$


By uniqueness

$$
M_{1}^{\mathbf{A}^{0}}(\lambda)=\bar{\tau}\left(M^{\mathbf{B}^{0}}\right)(-\bar{\lambda}) \tau
$$

and

$$
M_{1}^{\mathbf{A}^{0}}=-\bar{\tau}\left(M_{1}^{\mathbf{B}^{0}}\right) \tau
$$

Consequently, we have

$$
\mathcal{U}(x, t)=\left(\begin{array}{l}
\mathbf{u}(x, t) \\
\mathbf{v}(x, t)
\end{array}\right)=\frac{i}{\sqrt{12 \lambda_{0} t}}\left(M_{1}^{\mathbf{A}^{0}}-\sigma_{1}\left(\overline{M_{1}^{\mathbf{A}^{0}}}\right)\right)_{12}+O\left(\frac{\log t}{t}\right) .
$$

\subsection{The final transformation}

To solve for $\left(M_{1}^{\mathbf{A}^{0}}\right)_{12}$ explicitly, it is worthy considering the following transformation

$$
\Psi(\lambda)=H(\lambda)\left(-\frac{1}{\lambda}\right)^{i v \sigma} e^{i \lambda^{2} \sigma / 4}, \quad H(\lambda)=\left(\delta_{\mathbf{A}}\right)^{-\sigma} M^{\mathbf{A}^{0}}(\lambda)\left(\delta_{\mathbf{A}}\right)^{\sigma} .
$$

Therefore

$$
\Psi_{+}(\lambda)=\Psi_{-}(\lambda) v\left(-\lambda_{0}\right), \quad v=(-\lambda)^{i v \widehat{\sigma}} e^{-i \lambda^{2} \widehat{\sigma} / 4}\left(\delta_{\mathbf{A}}\right)^{-\widehat{\sigma}} J^{\mathbf{A}^{0}}
$$

Because of the jump matrix is independent of $\lambda$, we have

$$
\frac{d \Psi_{+}(\lambda)}{d \lambda}=\frac{d \Psi_{-}(\lambda)}{d k} v\left(-\lambda_{0}\right)
$$

Since $\frac{d \Psi(\lambda)}{d \lambda}$ and $\Psi$ have the same jump matrix along any of the rays, it follows that $\frac{d \Psi(\lambda)}{d \lambda} \Psi^{-1}(\lambda)$ is holomorphic in the complex plane and admits a polynomial dependence on $\lambda$ at $\lambda \rightarrow \infty$. In reality

$$
\begin{aligned}
\frac{d \Psi(\lambda)}{d \lambda} \Psi^{-1}(\lambda) & =\frac{i \lambda}{2} H(\lambda) \sigma H^{-1}(\lambda)-\frac{i v}{\lambda} H(\lambda) \sigma H^{-1}(\lambda)+\frac{d H(\lambda)}{d \lambda} H^{-1}(\lambda) \\
& =\frac{i \lambda}{2} \sigma-\frac{i}{2} \delta_{\mathbf{A}} \sigma\left[\sigma, M_{1}^{\mathbf{A}^{0}}\right] \delta_{\mathbf{A}}^{-\sigma}+O\left(\frac{1}{\lambda}\right) .
\end{aligned}
$$

Thus,

$$
\frac{d \Psi(\lambda)}{d \lambda}=\frac{i \lambda}{2} \sigma \Psi(\lambda)+\beta \Psi(\lambda),
$$

where

$$
\beta=-\frac{i}{2} \delta_{\mathbf{A}}^{\sigma}\left[\sigma, M_{1}^{\mathbf{A}^{0}}\right] \delta_{\mathbf{A}}^{-\sigma}=\left(\begin{array}{cc}
0 & \beta_{12} \\
\beta_{21} & 0
\end{array}\right)
$$

Particularly

$$
\left(M_{1}^{\mathbf{A}^{0}}\right)_{12}=i\left(\delta_{\mathbf{A}}\right)^{2} \beta_{12} .
$$


Let

$$
\Psi(\lambda)=\left(\begin{array}{ll}
\Psi_{11}(\lambda) & \Psi_{12}(\lambda) \\
\Psi_{21}(\lambda) & \Psi_{22}(\lambda)
\end{array}\right)
$$

It follows from 3.12 that

$$
\begin{aligned}
\frac{d^{2} \beta_{12} \Psi_{11}(\lambda)}{d \lambda^{2}} & =\left(\beta_{21} \beta_{12}+0.5 i-\frac{\lambda^{2}}{2}\right) \beta_{21} \Psi_{11}(\lambda), \\
\frac{d^{2} \Psi_{22}(\lambda)}{d \lambda^{2}} & =\left(\beta_{21} \beta_{12}-0.5 i-\frac{\lambda^{2}}{4}\right) \Psi_{22}(\lambda), \\
\beta_{21} \Psi_{21}(\lambda) & =\frac{1}{\beta_{12}}\left(\frac{d \beta_{21} \Psi_{11}(\lambda)}{d \lambda}-\frac{i \lambda}{2} \beta_{21} \Psi_{11}(\lambda)\right), \\
\beta_{21} \Psi_{12}(\lambda) & =\frac{d \Psi_{22}(\lambda)}{d \lambda}+\frac{i \lambda}{2} \Psi_{22}(\lambda) .
\end{aligned}
$$

As is well known that

$$
g(\zeta)=c_{1} D_{a}(\zeta)+c_{2} D_{a}(-\zeta)
$$

admits the solution of Weber's equation [11, 18, 37

$$
g^{\prime \prime}(\zeta)+\left(a+\frac{1}{2}-\frac{\zeta^{2}}{4}\right) g(\zeta)=0
$$

where $D_{a}(\zeta)$ is the standard parabolic-cylinder function, and admits the following relations

$$
\begin{gathered}
D_{a}^{\prime}(\zeta)+\frac{\zeta}{2} D_{a}(\zeta)-a D_{a-1}(\zeta)=0 \\
D_{a}( \pm \zeta)=\Gamma(a+1) e^{i \pi a / 2} D_{-a-1}( \pm i \zeta)+\frac{\Gamma e^{-i \pi a / 2}}{\sqrt{2 \pi}} D_{-1-a}(\mp i \zeta)
\end{gathered}
$$

We know that as $\zeta \rightarrow \infty 37$

$$
D_{a}(\zeta)= \begin{cases}\zeta^{a} e^{-\zeta^{2} / 4}\left(1+O\left(\frac{1}{\zeta^{2}}\right)\right), & |\arg \zeta|<\frac{3 \pi}{4} \\ \zeta^{a} e^{-\zeta^{2} / 4}\left(1+O\left(\frac{1}{\zeta^{2}}\right)\right)-\frac{\sqrt{2 \pi}}{\Gamma(-a)} e^{a \pi i+\zeta^{2} / 4}\left(1+O\left(\frac{1}{\zeta^{2}}\right)\right), & \frac{\pi}{4}<\arg \zeta<\frac{5 \pi}{4} \\ \zeta^{a} e^{-\zeta^{2} / 4}\left(1+O\left(\frac{1}{\zeta^{2}}\right)\right)-\frac{\sqrt{2 \pi}}{\Gamma(-a)} e^{-a \pi i+\zeta^{2} / 4}\left(1+O\left(\frac{1}{\zeta^{2}}\right)\right), & -\frac{5 \pi}{4}<\arg \zeta<-\frac{\pi}{4}\end{cases}
$$

where $\Gamma$ is the Gamma function.

Let $a=-i \beta_{21} \beta_{12}$, we have

$$
\begin{aligned}
\beta_{21} \Psi_{11}(\lambda) & =c_{1} D_{a}\left(e^{3 \pi i / 4} \lambda\right)+c_{2} D_{a}\left(e^{-\pi i / 4} \lambda\right), \\
\Psi_{22}(\lambda) & =c_{3} D_{-a}\left(e^{-3 \pi i / 4} \lambda\right)+c_{4} D_{-a}\left(e^{\pi i / 4} \lambda\right) .
\end{aligned}
$$

It follows from $\arg \lambda \in\left(\frac{3 \pi}{4}, \pi\right) \cup\left(-\pi,-\frac{3 \pi}{4}\right)$ and $\lambda \rightarrow \infty$ that

$$
\Psi_{11}(\lambda)(-\lambda)^{-i v} e^{-i \lambda^{2} / 4} \rightarrow \mathcal{I}, \quad \Psi_{22}(\lambda)(-\lambda)^{-i v} e^{i \lambda^{2} / 4} \rightarrow 1,
$$


and

$$
\beta_{21} \Psi_{11}(\lambda)=\beta_{21} e^{-\pi v / 4} D_{a}\left(e^{3 \pi i / 4} \lambda\right), \quad v=-\beta_{21} \beta_{12}, \quad \Psi_{22}(\lambda)=e^{-\pi v / 4} D_{-a}\left(e^{-3 \pi i / 4} \lambda\right) .
$$

Thus

$$
\begin{aligned}
\Psi_{21}(\lambda) & =\frac{\beta_{21} e^{-\pi v / 4}}{\beta_{21} \beta_{12}}\left(D_{a}^{\prime}\left(e^{3 \pi i / 4} \lambda\right)-\frac{i \lambda}{2} D_{a}\left(e^{3 \pi i / 4} \lambda\right)\right)=\beta_{21} e^{\pi(i+v) / 4} D_{a-1}\left(e^{3 \pi i / 4} \lambda\right), \\
\Psi_{22} & =e^{-\pi v / 4}\left(D_{-a}^{\prime}\left(e^{-3 \pi i / 4} \lambda\right)+\frac{i \lambda}{2} D_{-a}\left(e^{-3 \pi i / 4} \lambda\right)\right)=a e^{\pi(i-v) / 4} D_{-a-1}\left(e^{-3 \pi i / 4} \lambda\right) .
\end{aligned}
$$

As $\arg \lambda \in\left(\frac{\pi}{4}, \frac{3 \pi}{4}\right)$ and $\lambda \rightarrow \infty$, we have

$$
\Psi_{11}(\lambda)(-\lambda)^{i v} e^{-i \lambda^{2} / 4} \rightarrow \mathcal{I}, \quad \Psi_{22}(\lambda)(-\lambda)^{i v} e^{i \lambda^{2} / 4} \rightarrow 1,
$$

then

$$
\beta_{21} \Psi_{11}(\lambda)=\beta_{21} e^{3 \pi v / 4} D_{a}\left(e^{-\pi i / 4} \lambda\right), \quad \Psi_{22}(\lambda)=e^{-\pi v / 4} D_{-a}\left(e^{-3 \pi i / 4} \lambda\right) .
$$

So that

$$
\begin{aligned}
\Psi_{21}(\lambda) & =\frac{\beta_{21} e^{3 \pi v / 4}}{\beta_{21} \beta_{12}}\left(D_{a}^{\prime}\left(e^{-\pi i / 4} \lambda\right)-\frac{i \lambda}{2} D_{a}\left(e^{-\pi i / 4} \lambda\right)\right)=\beta_{21} e^{-3 \pi(i-v) / 4} D_{a-1}\left(e^{-\pi i / 4} \lambda\right), \\
\beta_{21} \Psi_{12}(\lambda) & =a e^{\pi(i-v) / 4} D_{-a-1}\left(e^{-3 \pi i / 4} \lambda\right) .
\end{aligned}
$$

Along the ray $\arg \lambda=3 \pi / 4$, we have

$$
\begin{gathered}
\Psi_{+}(\lambda)=\Psi_{-}(\lambda)\left(\begin{array}{cc}
\mathcal{I} & 0 \\
-\gamma\left(-\lambda_{0}\right) & 1
\end{array}\right), \\
\beta_{21} e^{\pi(i-v) / 4} D_{a-1}\left(e^{3 \pi i / 4} \lambda\right)=\beta_{21} e^{-3 \pi(i-v) / 4} D_{a-1}\left(e^{-\pi i / 4} \lambda\right)-\gamma\left(-\lambda_{0}\right) e^{-\pi v / 4} D_{-a}\left(e^{-3 \pi i / 4} \lambda\right), \\
D_{-a}\left(e^{-3 \pi i / 4} \lambda\right)=\frac{\Gamma(-a+1) e^{-\pi i a / 2}}{\sqrt{2 \pi}} D_{a-1}\left(e^{-\pi i / 4} \lambda\right)+\frac{\Gamma(-a+1) e^{\pi i a / 2}}{\sqrt{2 \pi}} D_{a-1}\left(e^{3 \pi i / 4} \lambda\right), \\
\beta_{21}=\Gamma(-a+1) e^{-\pi v / 2} e^{3 \pi i / 4} \gamma\left(-\lambda_{0}\right)=\frac{-v \Gamma(-i v) e^{\pi v / 2} e^{-3 \pi i / 4}}{\sqrt{2 \pi}} \gamma\left(-\lambda_{0}\right) .
\end{gathered}
$$

It is clear to see that $\Psi^{-1}(\lambda)$ and $\Psi^{\dagger}(\bar{\lambda})$ satisfy the same RHP. Due to the uniqueness, we get

$$
\Psi^{-1}(\lambda)=\Psi^{\dagger}(\bar{\lambda})
$$

and thus

$$
\beta_{12}=-\beta_{21}^{\dagger}=\frac{v \Gamma(i v) e^{\pi v / 2} e^{-\pi i / 4}}{\sqrt{2 \pi}} \sigma_{1} \gamma^{T}\left(\lambda_{0}\right) .
$$

It follows from $\beta_{21} \beta_{12}=-v$ and $\Gamma(-i v)=\bar{\Gamma}(i v)$ that

$$
\frac{v \Gamma(i v) e^{\pi v / 2}}{\sqrt{2 \pi}}=\frac{\sqrt{v}}{\left|\gamma\left(\lambda_{0}\right)\right|} .
$$

Summarizing the above analysis, the following theorem holds. 
Theorem 3.14. Let $\left(u_{0}, v_{0}\right)$ belong to the Schwartz space $\mathcal{S}(\mathbb{R})$. Then suppose $u(x, t)$, $v(x, t)$ can solve the CSS equation (1.2). As $x<0$ and $\left|\frac{x}{t}\right|$ is bounded, the solutions $u(x, t), v(x, t)$ admit the following leading asymptotics

$$
u(x, t)=\frac{u_{a s}(x, t)}{\sqrt{t}}+O\left(\frac{\log t}{t}\right), \quad v(x, t)=\frac{v_{a s}(x, t)}{\sqrt{t}}+O\left(\frac{\log t}{t}\right),
$$

where

$$
\begin{aligned}
u_{a s}(x, t) & =\frac{\sqrt{v}}{\sqrt{12 \lambda_{0}}\left|\gamma\left(\lambda_{0}\right)\right|}\left(\left|\gamma_{2}\left(\lambda_{0}\right)\right| e^{i\left(\phi+\arg \gamma_{2}\left(\lambda_{0}\right)\right)}+\left|\gamma_{1}\left(\lambda_{0}\right)\right| e^{-i\left(\phi+\arg \gamma_{1}\left(\lambda_{0}\right)\right)}\right), \\
v_{a s}(x, t) & =\frac{\sqrt{v}}{\sqrt{12 \lambda_{0}}\left|\gamma\left(\lambda_{0}\right)\right|}\left(\left|\gamma_{4}\left(\lambda_{0}\right)\right| e^{i\left(\phi+\arg \gamma_{4}\left(\lambda_{0}\right)\right)}+\left|\gamma_{3}\left(\lambda_{0}\right)\right| e^{-i\left(\phi+\arg \gamma_{3}\left(\lambda_{0}\right)\right)}\right), \\
\phi & =16 t \lambda_{0}^{3}+\arg \Gamma(i v)+v \log \left(192 \lambda_{0}^{3} t\right)+\frac{1}{\pi} \int_{-\lambda_{0}}^{\lambda_{0}} \log \left(\frac{1+|\gamma(\xi)|^{2}}{1+\left|\gamma\left(\lambda_{0}\right)\right|^{2}}\right) \frac{d \xi}{\xi+\lambda_{0}}-\frac{5 \pi}{4}, \\
v & =\frac{1}{2 \pi} \log \left(1+\mid \gamma\left(\left.\lambda_{0}\right|^{2}\right),\right.
\end{aligned}
$$

and $\gamma_{1}, \gamma_{2}, \gamma_{3}, \gamma_{4}$ are the $1,2,3,4$-th component of the vector function $\gamma$ given by (2.17), $\lambda_{0}=\sqrt{-x /(12 t)}, \Gamma$ is a Gamma function.

\section{Conclusions and discussions}

In this work, we have obtained a $5 \times 5$ matrix Riemann-Hilbert problem to tackle the Cauchy problem for the CSS equation $(1.2)$ on the line, which can help us to obtain a representation for the solution of the CSS equation (1.2). We then employ the approach of the Deift-Zhou steepest descent to discuss the long-time asymptotics of the CSS equation (1.2). Similarly to [44], starting from the CSS equation (1.2), if we impose the following constraint

$$
v(x, t)=\bar{u}(-x, t)
$$

we then obtain

$$
u_{t}+\left\{u_{x x x}+6\left(|u|^{2}+|u(-x, t)|^{2}\right) u_{x}+3 u\left(|u|^{2}+|u(-x, t)|^{2}\right)_{x}\right\}=0
$$

which is a nonlocal equation of reverse-time type. If we impose the solution constraint

$$
v(x, t)=\bar{u}(-x,-t)
$$

the coupled Sasa-Satsuma 1.2 reduces to

$$
u_{t}+\left\{u_{x x x}+6\left(|u|^{2}+|u(-x,-t)|^{2}\right) u_{x}+3 u\left(|u|^{2}+|u(-x,-t)|^{2}\right)_{x}\right\}=0,
$$

which is a nonlocal equation of reverse-space-time type. The two equations differ from the other nonlocal equations of reverse-time and reverse-space-time types [2,15] in the 
nonlinear terms. Both of our nonlocal equations 4.1 and 4.2 are also integrable which have clear physical meanings.

Finally, we state that there exist several methods to derive exact solutions for the nonlinear wave equations, such as Darboux transformation, Inverse scattering transform, Riemann-Hilbert approach, Deift-Zhou steepest descent method, Hirota method, dressing method, Wronskian technique etc. Consequently, it is very worthy to consider whether the nonlocal equations (4.1) and (4.2) can be solved by using these approaches? These ideas will be left for future discussions.

\section{Acknowledgments}

We express our sincere thanks to the editor and reviewer for their valuable comments. This work is supported by the National Natural Science Foundation of China under Grant No. 11871180.

\section{References}

[1] M. J. Ablowitz and A. S. Fokas, Complex Variables: Introduction and applications, Second edition, Cambridge Texts in Applied Mathematics, Cambridge University Press, Cambridge, 2003.

[2] M. J. Ablowitz and Z. H. Musslimani, Integrable nonlocal nonlinear equations, Stud. Appl. Math. 139 (2017), no. 1, 7-59.

[3] R. Beals and R. R. Coifman, Scattering and inverse scattering for first order systems, Comm. Pure Appl. Math. 37 (1984), no. 1, 39-90.

[4] G. Biondini and D. Mantzavinos, Long-time asymptotics for the focusing nonlinear Schrödinger equation with nonzero boundary conditions at infinity and asymptotic stage of modulational instability, Comm. Pure Appl. Math. 70 (2017), no. 12, 23002365 .

[5] A. Boutet de Monvel, A. Its and V. Kotlyarov, Long-time asymptotics for the focusing NLS equation with time-periodic boundary condition on the half-line, Comm. Math. Phys. 290 (2009), no. 2, 479-522.

[6] P. Deift and X. Zhou, A steepest descent method for oscillatory Riemann-Hilbert problems: Asymptotics for the MKdV equation, Ann. of Math. (2) 137 (1993), no. 2, $295-368$. 
[7] _ Long-time asymptotics for solutions of the NLS equation with initial data in a weighted Sobolev space, Comm. Pure Appl. Math. 56 (2003), no. 8, 1029-1077.

[8] A. S. Fokas, A unified transform method for solving linear and certain nonlinear PDEs, Proc. Roy. Soc. London Ser. A 453 (1997), no. 1962, 1411-1443.

[9] A. S. Fokas, A. R. Its and L.-Y. Sung, The nonlinear Schrödinger equation on the half-line, Nonlinearity 18 (2005), no. 4, 1771-1822.

[10] A. S. Fokas and J. Lenells, The unified method I: Nonlinearizable problems on the half-line, J. Phys. A 45 (2012), no. 19, 195201, 38 pp.

[11] X. Geng and H. Liu, The nonlinear steepest descent method to long-time asymptotics of the coupled nonlinear Schrödinger equation, J. Nonlinear Sci. 28 (2018), no. 2, 739-763.

[12] K. Grunert and G. Teschl, Long-time asymptotics for the Korteweg-de Vries equation via nonlinear steepest descent, Math. Phys. Anal. Geom. 12 (2009), no. 3, 287-324.

[13] B. Guo and N. Liu, Long-time asymptotics for the Kundu-Eckhaus equation on the half-line, J. Math. Phys. 59 (2018), no. 6, 061505, 19 pp.

[14] B. Guo, N. Liu and Y. Wang, Long-time asymptotics for the Hirota equation on the half-line, Nonlinear Anal. 174 (2018), 118-140.

[15] J.-L. Ji and Z.-N. Zhu, Soliton solutions of an integrable nonlocal modified Kortewegde Vries equation through inverse scattering transform, J. Math. Anal. Appl. 453 (2017), no. 2, 973-984.

[16] J. Lenells, Initial-boundary value problems for integrable evolution equations with $3 \times 3$ Lax pairs, Phys. D 241 (2012), no. 8, 857-875.

[17] H. Liu, X. Geng and B. Xue, The Deift-Zhou steepest descent method to long-time asymptotics for the Sasa-Satsuma equation, J. Differential Equations 265 (2018), no. $11,5984-6008$.

[18] N. Liu and B. Guo, Long-time asymptotics for the Sasa-Satsuma equation via nonlinear steepest descent method, J. Math. Phys. 60 (2019), no. 1, 011504, 25 pp.

[19] W.-X. Ma, Riemann-Hilbert problems and $N$-soliton solutions for a coupled $m K d V$ system, J. Geom. Phys. 132 (2018), 45-54.

[20] _ Application of the Riemann-Hilbert approach to the multicomponent AKNS integrable hierarchies, Nonlinear Anal. Real World Appl. 47 (2019), 1-17. 
[21] _ The inverse scattering transform and soliton solutions of a combined modified Korteweg-de Vries equation, J. Math. Anal. Appl. 471 (2019), no. 1-2, 796-811.

[22] _ Long-time asymptotics of a three-component coupled mKdV system, Mathematics 7 (2019), no. 7, 573-610.

[23] A. Mahalingam and K. Porsezian, Propagation of dark solitons in a system of coupled higher-order nonlinear Schrödinger equations, J. Phys. A 35 (2002), no. 13, 30993109 .

[24] K. Nakkeeran, K. Porsezian, P. Shanmugha Sundaram and A. Mahalingam, Optical solitons in $N$-coupled higher order nonlinear Schrödinger equations, Phys. Rev. Lett. 80 (1998), no. 7, 1425-1428.

[25] J. J. C. Nimmo and H. Yilmaz, Binary Darboux transformation for the Sasa-Satsuma equation, J. Phys. A 48 (2015), no. 42, 425202, 16 pp.

[26] N. Sasa and J. Satsuma, New-type of soliton solutions for a higher-order nonlinear Schrödinger equation, J. Phys. Soc. Japan 60 (1991), no. 2, 409-417.

[27] S.-F. Tian, The mixed coupled nonlinear Schrödinger equation on the half-line via the Fokas method, Proc. A. 472 (2016), no. 2195, 20160588, 22 pp.

[28] _ Initial-boundary value problems for the general coupled nonlinear Schrödinger equation on the interval via the Fokas method, J. Differential Equations 262 (2017), no. $1,506-558$.

[29] S.-F. Tian and T.-T. Zhang, Long-time asymptotic behavior for the Gerdjikov-Ivanov type of derivative nonlinear Schrödinger equation with time-periodic boundary condition, Proc. Amer. Math. Soc. 146 (2018), no. 4, 1713-1729.

[30] M. N. Vinoj and V. C. Kuriakose, Multisoliton solutions and integrability aspects of coupled higher-order nonlinear Schrödinger equations, Phys. Rev. E (3) 62 (2000), no. 6, part B, 8719-8725.

[31] D.-S. Wang, B. Guo and X. Wang, Long-time asymptotics of the focusing KunduEckhaus equation with nonzero boundary conditions, J. Differential Equations 266 (2019), no. 9, 5209-5253.

[32] D.-S. Wang, D.-J. Zhang and J. Yang, Integrable properties of the general coupled nonlinear Schrödinger equations, J. Math. Phys. 51 (2010), no. 2, 023510, 17 pp. 
[33] X.-B. Wang and B. Han, The three-component coupled nonlinear Schrödinger equation: Rogue waves on a multi-soliton background and dynamics, Europhys. Lett. 126 (2019), no. 1, 15001, 6 pp.

[34] _ The pair-transition-coupled nonlinear Schrödinger equation: The RiemannHilbert problem and N-soliton solutions, Eur. Phys. J. Plus 134 (2019), no. 2, 78.

[35] _ Long-time behavior for the Cauchy problem of the 3-component Manakov system, Phys. Scr. 95 (2020), no. 6, 065226, 13 pp.

[36] _ A Riemann-Hilbert approach to a generalized nonlinear Schrödinger equation on the quarter plane, Math. Phys. Anal. Geom. 23 (2020), no. 2, Paper No. 25, 23 pp.

[37] E. T. Whittaker and G. N. Watson, A Course of Modern Analysis, Reprint of the fourth (1927) edition, Cambridge Mathematical Library, Cambridge University Press, Cambridge, 1996.

[38] J.-P. Wu and X.-G. Geng, Inverse scattering transform of the coupled Sasa-Satsuma equation by Riemann-Hilbert approach, Commun. Theor. Phys. (Beijing) 67 (2017), no. $5,527-534$.

[39] J. Xu and E. Fan, The unified transform method for the SasaSatsuma equation on the half-line, Proc. R. Soc. Lond. Ser. A Math. Phys. Eng. Sci. 469 (2013), no. 2159, 20130068, 25 pp.

[40] _ Long-time asymptotics for the Fokas-Lenells equation with decaying initial value problem: Without solitons, J. Differential Equations 259 (2015), no. 3, 10981148.

[41] _ Initial-boundary value problem for integrable nonlinear evolution equation with $3 \times 3$ Lax pairs on the interval, Stud. Appl. Math. 136 (2016), no. 3, 321-354.

[42] J. Xu, E. Fan and Y. Chen, Long-time asymptotic for the derivative nonlinear Schrödinger equation with step-like initial value, Math. Phys. Anal. Geom. 16 (2015), no. $3,253-288$.

[43] T. Xu and X.-M. Xu, Single- and double-hump femtosecond vector solitons in the coupled Sasa-Satsuma system, Phys. Rev. E 87 (2013), 032913, 6 pp.

[44] J. Yang, Physically significant nonlocal nonlinear Schrödinger equation and its soliton solutions, Phys. Rev. E 98 (2018), no. 4, 042202, 12 pp. 
[45] J. Yang and D. J. Kaup, Squared eigenfunctions for the Sasa-Satsuma equation, J. Math. Phys. 50 (2009), no. 2, 023504, 21 pp.

Xiu-Bin Wang and Bo Han

Department of Mathematics, Harbin Institute of Technology, Harbin 150001, China E-mail addresses: xiubinwang@163.com, bohan@hit.edu.cn 